\title{
Performance Analysis of OFDM-Based Decode-and-Forward Relay Network with Multiple Interferers over Rayleigh Fading Channels
}

\author{
Jia Tu, ${ }^{1}$ Yueming Cai, ${ }^{1,2}$ and Weiwei Yang1 \\ ${ }^{1}$ Institute of Communications Engineering, PLA University of Science and Technology, Nanjing 210007, China \\ ${ }^{2}$ National Mobile Communications Research Laboratory, Southeast University, Nanjing 210096, China \\ Correspondence should be addressed to Jia Tu, tujia_666@163.com
}

Received 1 June 2010; Revised 19 August 2010; Accepted 8 October 2010

Academic Editor: Robert Schober

Copyright () 2011 Jia Tu et al. This is an open access article distributed under the Creative Commons Attribution License, which permits unrestricted use, distribution, and reproduction in any medium, provided the original work is properly cited.

We consider an OFDM-based decode-and-forward relay network with multiple uncorrelated equal power interferers over frequency-selective Rayleigh fading channels and derive a unified closed-form expression of average symbol-error ratio for $M$ ary phase-shift-keying (M-PSK) modulation. Simulations are carried out to validate our analyses.

\section{Introduction}

Relay communications, as a promising technique to help in attaining broader coverage and combating the impairments of the wireless channel, have gained significant interests at the beginning of this century [1-3]. Recently, various cooperation protocols, for example, amplify-and-forward $(\mathrm{AF})$ and decode-and-forward (DF) [2], have been proposed for cooperative wireless networks. Subsequently, the performance analysis of the relay network has attracted a lot of interest, considering different issues, such as cooperation protocols [4], channel models [5], power allocation schemes [6], and so on.

Due to aggressive reusing of frequency channels for highspectrum utilization, the cochannel interference (CCI) is unavoidable in the cellular system. By now, many excellent works have investigated the performance of the traditional point-to-point wireless system in the presence of CCI [7-9]. However, in the relay network, the CCI also appears, due to reusing the same frequency channel for both the competing users and the relays. In [10], the authors examine the feasibility of applying collaborative relays to the large-scale wireless network for the throughput improvement by modeling the interference-sensitive region. In [11], the authors consider a time-division multiple-access (TDMA) system in which a single time slot is shared by many relays and employ dual-hop relays to improve its throughput. Based on the model in [11], the authors in [12] consider the case where the destination is corrupted by a number of CCIs, while the relay is only perturbed by an additive white Gaussian noise (AWGN) and obtain the closed-form expression of the outage probability for the dual-hop relay system.

It should be pointed out that all the aforementioned papers are confined to the single relay, the flat-fading channel, or the special case in which the interference only affects the destination node. In practice, most wireless transmissions experience the scattered and delayed propagation paths, which result in the so-called frequency-selective fading channels. For multiple relays and frequency-selective fading channels, more should be considered involving the selection of the relays, the combiner for combating multiple interferers at the relays and the destination, and so on.

For frequency-selective channels, the orthogonal frequency division multiplexing (OFDM) is often employed in practice. Hence, many works which have been provided are focusing on OFDM-based relay networks [13-15]. It remains an open problem to determine the performance of OFDM-based relay networks over frequency-selective fading channels. The performance of OFDM-based relay networks is a significative work for the system design.

Main Contribution. In this paper, we consider an OFDMbased DF relay network over frequency-selective Rayleigh fading channels. The optimum combining (OC) [7-9] is 


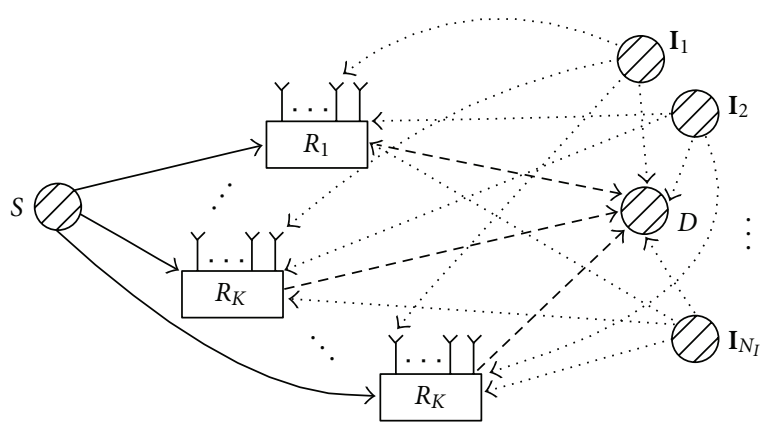

$\longrightarrow$ Phase I

$--\rightarrow$ Phase II

$\cdots>$ Interferer

FIGURE 1: System model.

employed to combat multiple uncorrelated equal power interferers at the relays and the destination. Based on the work of [16], we derive an unified closed-form expression of average SER for this relay network with M-PSK modulation.

The paper is organized as follows. Section 2 describes the system and channel models. The performance analysis is presented in Section 3. In Section 4, simulation results are given. Finally, some conclusions are drawn in Section 5.

Notation. The $N \times N$ identity matrix is denoted by $\mathbf{I}_{N}$. $C N(0, \mathbf{R})$ denotes a circularly symmetric complex normal zero mean random vector with covariance matrix R. $(\bullet)^{T}$ and $(\bullet)^{H}$ denote the transpose and conjugate transpose of a vector/matrix, respectively.

\section{System Model}

In this paper, we consider a half-duplex relay network depicted in Figure 1, which is composed of one source node $S$, one destination node $D$, and a set of $K$ relays $S_{R}=$ $\left\{R_{1}, \ldots, R_{K}\right\}$. Both the source node and the destination node are equipped with one antenna, while each relay has $L$ antennas in order to combat the multiple interferers in the network, but only one antenna is utilized at each relay for forwarding. In fact, such system architecture is also discussed in $[17,18]$. The relay nodes, which are fixed, are located between the source and the destination. It is assumed that there is no direct link between the source and the destination because of long distance or obstacles. An OFDM transceiver with $N_{\text {sub }}$ subcarriers is available at each node. Perfect time and frequency synchronization among nodes are assumed. We also assume that a cyclic prefix (CP) is long enough to accommodate the channel delay spread.

We adopt an OFDM-based DF relaying strategy, and the transmission is divided into two distinct phases. In the first phase, the source node broadcasts the OFDM symbols to the $K$ relays. In the second phase, the relays, which can decode the signals properly (judged by the signal-to-noise ratio (SNR) threshold [19]), retransmit the corresponding subcarriers to the destination in a timedivision-multiplex (TDM) mode. We consider that there are other $N_{I}$ sources (e.g., other users in the cellular network) as distinct interference sources in the relay network, and these interferers affect both the received signals at the relays and the destination (as Figure 1). Under such conditions, OC, which is a well-known method to combat fading and suppress the CCI in wireless communication systems with reception diversity, is employed to maximize the signal-tointerference plus noise ratio (SINR) at the relays and the destination.

In order to simplify the analysis, we suppose that each relay uses one antenna to retransmit the signal, and $K^{\prime}\left(K^{\prime} \in\right.$ $[0, K])$ relays can properly decode the received signal from the source node among $K$ relays. Further, the fading channels from any node to the lth antenna of the $k$ th relay node or the destination node are all assumed to be quasistatic frequency-selective fading; that is, they do not change within one OFDM symbol, but vary from one symbol to another. In this paper, $\hat{\mathbf{h}}_{S R_{k, l}}=\left[\hat{h}_{S R_{k, l}}(0), \ldots, \hat{h}_{S R_{k, l}}\left(L_{\text {tap }}-1\right)\right]^{T}$ and $\hat{\mathbf{h}}_{I_{m} R_{k, l}}=\left[\hat{h}_{I_{m} R_{k, l}}(0), \ldots, \hat{h}_{I_{m} R_{k, l}}\left(L_{\mathrm{tap}}-1\right)\right]^{T}$ are assumed to be the channel impulse response (CIR) vectors from the source node and the $m$ th interferer to the $l$ th antenna of the $k$ th relay node, respectively, while $\hat{\mathbf{h}}_{R_{k} D}=\left[\hat{h}_{R_{k} D}(0), \ldots, \hat{h}_{R_{k} D}\left(L_{\text {tap }}-\right.\right.$ 1) $]^{T}$ and $\hat{\mathbf{h}}_{I_{m} D_{k}}=\left[\hat{h}_{I_{m} D_{k}}(0), \ldots, \hat{h}_{I_{m} D_{k}}\left(L_{\text {tap }}-1\right)\right]^{T}$ are the CIR vectors from the $k$ th relay and $m$ th interferer to the destination node at the subslot of $k$ th relay node retransmitting, respectively, where $L_{\text {tap }}$ represents the number of the taps of the corresponding channel. Each element of $\hat{\mathbf{h}}_{S_{k, l},}, \hat{\mathbf{h}}_{I_{m} R_{k, l}}, \hat{\mathbf{h}}_{R_{k} D}$, and $\hat{\mathbf{h}}_{I_{m} D_{k}}$ is modeled as a zero-mean complex Gaussian random variable with variance $\sigma_{x, l}^{2}, x \in$ $\left\{S R_{k, l}, I_{m} R_{k, l}, R_{k} D, \quad I_{m} D, l=1, \ldots, L, \quad m=1, \ldots, N_{I}, \quad k=\right.$ $1, \ldots, K\}$.

In the first phase, the source node broadcasts the OFDM symbols to the $K$ relays, while each relay is corrupted by a number of CCIs. Denote $s_{S}=\left[s_{S}(0), \ldots, s_{S}\left(N_{\text {sub }}-1\right)\right]^{T}$ as the frequency-domain symbol transmitted by the source node and $\mathbf{s}_{I_{m}}=\left[s_{I_{m}}(0), \ldots, s_{I_{m}}\left(N_{\text {sub }}-1\right)\right]^{T}$ as the frequencydomain interference symbol of the $m$ th interferer normalized such that $P_{S}$ and $P_{I_{m}}$ represent their corresponding transmitting powers, respectively. The CP is added to the top of each signal vector after taking IFFT, respectively. Consider that the $k$ th $(k \in[1, K])$ relay receiver provides space diversity via an $L$-element antenna array, and assume that the antenna elements of the array are placed sufficiently far apart so as to provide independent fading paths. Here, we assume that all interference sources have equal transmitting powers $P_{I} \triangleq P_{I_{1}}=\cdots=P_{I_{N_{I}}}$, and the interference $\operatorname{signal}_{I_{m}}(n)$ is Gaussian distributed with zero mean and unit variance. This assumption is widely used in the literature, as in [8]. Thus, the received time-domain signal after moving the $\mathrm{CP}$ at the $l$ th antenna of $k$ th relay can be expressed as

$$
\hat{\mathbf{y}}_{r_{k, l}}=\sqrt{P_{S}} \hat{\mathbf{H}}_{S R_{k, l}} \mathbf{F}^{H} \boldsymbol{s}_{S}+\sqrt{P_{I}} \sum_{m=1}^{N_{I}} \hat{\mathbf{H}}_{I_{m} R_{k, l}} \mathbf{F}^{H} \boldsymbol{s}_{I_{m}}+\widehat{\mathbf{n}}_{r_{k, l},}
$$


where $\hat{\mathbf{H}}_{S R_{k, l}}$ and $\hat{\mathbf{H}}_{I_{m} R_{k, l}}$ are two $N_{\text {sub }} \times N_{\text {sub }}$ circulant matrices with $\left[\mathbf{h}_{S R_{k, l},}, \mathbf{0}_{1 \times\left(N_{\text {sub }}-L_{\text {tap }}-1\right)}\right]^{T}$ and $\left[\mathbf{h}_{I_{m} R_{k, l},}, \mathbf{0}_{1 \times\left(N_{\text {sub }}-L_{\text {tap }}-1\right)}\right]^{T}$ as their first columns, respectively, $\mathbf{F}$ is the unitary discrete Fourier transform matrix with $[\mathbf{F}]_{p, q}=1 / \sqrt{N_{\text {sub }}} e^{-j 2 \pi p q / N_{\text {sub }}}$, and $\hat{\mathbf{n}}_{r_{k, l}}$ is an AWGN vector with zero mean and covariance $\operatorname{matrix} E\left\{\hat{\mathbf{n}}_{r_{k, l}} \hat{\mathbf{n}}_{r_{k, l}}^{H}\right\}=\sigma^{2} \mathbf{I}_{N_{\text {sub }}}$.

After taking FFT to $\hat{\mathbf{y}}_{r_{k, l}}$, the received frequency-domain signal at the $l$ th antenna of $k$ th relay can be written as follows:

$$
\begin{aligned}
\mathbf{y}_{r_{k, l}} & =\sqrt{P_{S}} \mathbf{F} \hat{\mathbf{H}}_{S R_{k, l}} \mathbf{F}^{H} \mathbf{s}_{S}+\sqrt{P_{I}} \sum_{m=1}^{N_{I}} \hat{\mathbf{F}} \hat{\mathbf{H}}_{I_{m} R_{k, l}} \mathbf{F}^{H} \mathbf{s}_{I_{m}}+\mathbf{F} \hat{\mathbf{n}}_{r_{k, l}} \\
& =\sqrt{P_{S}} \mathbf{H}_{S R_{k, l}} \mathbf{s}_{S}+\sqrt{P_{I}} \sum_{m=1}^{N_{I}} \mathbf{H}_{I_{m} R_{k, l}} \mathbf{s}_{I_{m}}+\mathbf{n}_{r_{k, l},},
\end{aligned}
$$

where $\mathbf{n}_{r_{k, l}}$ is an AWGN vector with zero mean and covariance matrix $E\left\{\mathbf{n}_{r_{k, l}} \mathbf{n}_{r_{k, l}}^{H}\right\}=\sigma^{2} \mathbf{I}_{N_{\text {sub }}}, \mathbf{H}_{S R_{k, l}}=\hat{\mathbf{F H}}_{S R_{k, l}} \mathbf{F}^{H}, \mathbf{H}_{I_{m} R_{k, l}}=$ $\hat{\mathbf{F H}}_{I_{m} R_{k, l}} \mathbf{F}^{H}$. Under the assumption that $\mathrm{CP}$ is long enough, we have $\mathbf{H}_{S R_{k, l}}=\operatorname{diag}\left(h_{S R_{k, l}}(0), h_{S R_{k, l}}(1), \ldots, h_{S R_{k, l}}\left(N_{\text {sub }}-1\right)\right)$ and $\mathbf{H}_{I_{m} R_{k, l}}=\operatorname{diag}\left(h_{I_{m} R_{k, l}}(0), h_{I_{m} R_{k, l}}(1), \ldots, h_{I_{m} R_{k, l}}\left(N_{\text {sub }}-1\right)\right)$, where $h_{x}(n)=\sum_{p=0}^{L_{\text {tap }}} \hat{h}_{x}(p) e^{-j 2 \pi n p / N_{\text {sub }}}, x \in\left\{S R_{k, l}, I_{m} R_{k, l}\right.$, $\left.l=1, \ldots, L, m=1, \ldots, N_{I}, k=1, \ldots, K\right\}$ is the channelfrequency response on the $n$th subcarrier of corresponding channel. From (2), it can be found that when the CP is long enough to accommodate the channel delay spread and the channel is quasistatic frequency-selective fading, these subcarriers are orthogonal to each other absolutely, and the transmission on each subcarrier is parallel. For the sake of simplicity, we focus the performance of this system at the $n$th subcarrier.

Associate with $L$ antennas of the $k$ th relay node, and denote the channel response vectors of the $n$th subcarrier from the source and the $m$ th interference node to the $k$ th relay node as $\mathbf{h}_{S R_{k}}(n)=\left[h_{S R_{k, 1}}(n), \ldots, h_{S R_{k, L}}(n)\right]^{T}$ and $\mathbf{h}_{I_{m} R_{k}}(n)=\left[h_{I_{m} R_{k, 1}}(n), \ldots, h_{I_{m} R_{k, L}}(n)\right]^{T}$, respectively. The complex array output vector at the $k$ th relay can be written as

$$
\mathbf{y}_{r_{k}}(n)=\sqrt{P_{S}} \mathbf{h}_{S R_{k}}(n) \mathcal{S}_{S}(n)+\sum_{m=1}^{N_{I}} \sqrt{P_{I_{m}}} \mathbf{h}_{I_{m} R_{k}}(n) \mathcal{S}_{I_{m}}(n)+\mathbf{n}_{r_{k}}(n),
$$

where $\mathbf{n}_{r_{k}}(n)$ represents the AWGN with zero mean and variance $\sigma^{2}$.

In order to combat the interferers, some alternatives can be used at the receiver, such as minimum mean-square error (MMSE) [20] and OC. In [20], the authors prove that MMSE and $\mathrm{OC}$ receivers are equivalent. We, however, focus on the OC receiver in this paper due to the feasibility of the analysis.

At the $k$ th relay, the weights are selected to maximize the instantaneous SINR $\gamma_{k}(n)$ at the combiner, and thus $\mathbf{w}_{k}(n)=$ $\mathbf{R}_{n I_{k}}^{-1}(n) \mathbf{h}_{S R_{k}}(n)$, where $\mathbf{R}_{n I_{k}}(n)$ is the noise-plus-interference covariance matrix of the $n$th subcarrier

$$
\begin{aligned}
\mathbf{R}_{n I_{k}}(n)= & E\left\{\left(\sum_{m=1}^{N_{I}} \sqrt{P_{I}} \mathbf{h}_{I_{m} R_{k}}(n) s_{I_{m}}(n)+\mathbf{n}_{r_{k}}(n)\right)\right. \\
& \left.\times\left(\sum_{n=1}^{N_{I}} \sqrt{P_{I}} \mathbf{h}_{I_{m} R_{k}}(n) s_{I_{m}}(n)+\mathbf{n}_{r_{k}}(n)\right)^{H}\right\} \\
= & P_{I} \sum_{m=1}^{N_{I}} \mathbf{h}_{I_{m} R_{k}}(n) \mathbf{h}_{I_{m} R_{k}}^{H}(n)+\sigma^{2} \mathbf{I}_{L} .
\end{aligned}
$$

For the OC receiver, $\gamma_{k}(n)$ is given by [21]

$$
\gamma_{k}(n)=P_{S} \mathbf{h}_{S R_{k}}^{H}(n) \mathbf{R}_{n I_{k}}^{-1}(n) \mathbf{h}_{S R_{k}}(n)=\sum_{l=1}^{L} \frac{\sigma^{2}}{\lambda_{k, l}(n)} \gamma_{k, l}(n),
$$

where $\gamma_{k, l}(n) \triangleq\left|h_{S R_{k, l}}(n)\right|^{2} P_{S} / \sigma^{2}$ is the instantaneous SNR of $l$ th antenna, $\lambda_{k, 1}(n), \ldots, \lambda_{k, L}(n)$ are the eigenvalues of $\mathbf{R}_{n I_{k}}(n)$.

In the second phase, the $k$ th relay checks whether its decoded symbols are correct. As we know, different ways can be used to do the judgment, such as using cyclic redundancy code (CRC) [22] or utilizing SNR threshold. In our paper, we use the SNR threshold method. That is to say, after OC, the relay checks the SNR of the received signal against a preset threshold. The relay decodes and forwards only when this SNR is greater than the threshold. Suppose that $K^{\prime}$ $\left(K^{\prime} \in[0, K]\right)$ relays, which are $R_{z_{1}}, R_{z_{2}}, \ldots, R_{z_{K^{\prime}}}$, can be selected to retransmit the decoded symbols using the SNR threshold, so the set of these relays can be denoted as $S_{1}=$ $\left\{R_{z_{1}}, R_{z_{2}}, \ldots, R_{z_{K^{\prime}}}\right\}$. Then, these $K^{\prime}$ relays will retransmit the information to the destination in the TDM mode. Although the relays have been equipped with $L$ antennas, we consider that each relay uses one antenna to send the signal to the destination. As mentioned in [17], a single transmitting antenna is adopted to keep the cost comparable to a conventional single-antenna relay network, where one receiver chain is required. Similar to the channel from the source to the $k$ th relay in (1), there are $N_{I}$ interferers between each selected relay and the destination. At the destination, the time-domain received signal from $k$ th relay $\left(R_{k} \in S_{1}\right)$ is

$$
\hat{\mathbf{y}}_{D_{k}}=\sqrt{P_{R_{k}}} \hat{\mathbf{H}}_{R_{k} D} \mathbf{F}^{H} \mathbf{s}_{S}+\sqrt{P_{I}} \sum_{m=1}^{N_{I}} \hat{\mathbf{H}}_{I_{m} D_{k}} \mathbf{F}^{H} \mathbf{s}_{I_{m}}+\widehat{\mathbf{n}}_{D_{k}},
$$

where $P_{R_{k}}$ represents the transmitting power at the $k$ th relay, and $\hat{\mathbf{n}}_{D_{k}}$ represents an AWGN vector with zero mean and covariance matrix $\sigma^{2} \mathbf{I}_{N_{\text {sub }}}$ at the destination. $\hat{\mathbf{H}}_{R_{k} D}$ and $\hat{\mathbf{H}}_{I_{m} D_{k}}$ are two $N_{\text {sub }} \times N_{\text {sub }}$ circulant matrices with $\left[\hat{\mathbf{h}}_{R_{k} D}, \mathbf{0}_{1 \times\left(N_{\text {sub }}-L_{\text {tap }}-1\right)}\right]^{T}$ and $\left[\mathbf{h}_{I_{m} D_{k}}, \mathbf{0}_{1 \times\left(N_{\text {sub }}-L_{\text {tap }}-1\right)}\right]^{T}$ as their first columns, respectively. 
Taking FFT to $\hat{\mathbf{y}}_{D_{k}}$, the received frequency-domain signal at the destination is

$$
\begin{aligned}
\mathbf{y}_{D_{k}} & =\sqrt{P_{R_{k}}} \hat{\mathbf{H}}_{R_{k} D} \mathbf{F}^{H} \mathbf{s}_{S}+\sqrt{P_{I}} \sum_{m=1}^{N_{I}} \hat{\mathbf{F H}} \hat{\mathbf{H}}_{I_{m} D_{k}} \mathbf{F}^{H} \mathbf{s}_{I_{m}}+\mathbf{F} \hat{\mathbf{n}}_{D_{k}} \\
& =\sqrt{P_{S}} \mathbf{H}_{R_{k} D} \mathbf{s}_{S}+\sqrt{P_{I}} \sum_{m=1}^{N_{I}} \mathbf{H}_{I_{m} D_{k}} \mathbf{s}_{I_{m}}+\mathbf{n}_{D_{k}},
\end{aligned}
$$

where $\mathbf{n}_{D_{k}}$ is an AWGN vector with zero mean and covariance matrix $\sigma^{2} \mathbf{I}_{N_{\text {sub }}} \cdot \mathbf{H}_{R_{k} D}=\mathbf{F H}_{R_{k} D} \mathbf{F}^{H}=$ $\operatorname{diag}\left(h_{R_{k} D}(0), \ldots, h_{R_{k} D}\left(N_{\text {sub }}-1\right)\right)$ and $\mathbf{H}_{I_{m} D_{k}}=$ $\mathbf{F H}_{I_{m} D_{k}} \mathbf{F}^{H}=\operatorname{diag}\left(h_{I_{m} D_{k}}(0), \ldots, h_{I_{m} D_{k}}\left(N_{\text {sub }}-1\right)\right)$, where $h_{x}(n)=\sum_{p=0}^{L_{\text {tap }}} \hat{h}_{x}(p) e^{-j 2 \pi n p / N_{\text {sub }}}, x \in\left\{R_{k} D, I_{m} D_{k}, m=\right.$ $\left.1, \ldots, N_{I}, k=1, \ldots, K\right\}$ is the channel frequency response on the $n$th subcarrier of corresponding channel.

After receiving the signals from all $K^{\prime}$ relays, the OC is employed by the destination to obtain the final signal. Similar to the first phase, we denote the CIR vectors of the $n$th subcarrier from the selected relays and the $m$ th interferer to the destination node as $\mathbf{h}_{R D}(n)=\left[h_{R_{1} D}(n), \ldots, h_{R_{K^{\prime}} D}(n)\right]^{T}$ and $\mathbf{h}_{I_{m} D}(n)=\left[h_{I_{m} D_{1}}(n), \ldots, h_{I_{m} D_{K^{\prime}}}(n)\right]^{T}$, respectively. Let $\sqrt{\mathbf{P}_{R}}=$ $\operatorname{diag}\left(\sqrt{P_{R_{1}}}, \ldots, \sqrt{P_{R_{K^{\prime}}}}\right)$, the output vector at the destination is

$$
\mathbf{y}_{D}(n)=\sqrt{\mathbf{P}_{R}} \mathbf{h}_{R D}(n) s_{S}(n)+\sqrt{P_{I}} \sum_{m=1}^{N_{I}} \mathbf{h}_{I_{m} D}(n) s_{I_{m}}(n)+\mathbf{n}_{D}(n),
$$

where $\mathbf{y}_{D}(n)=\left[y_{D_{1}}(n), \ldots, y_{D_{K^{\prime}}}(n)\right]^{T}$ and $\mathbf{n}_{D}(n)=\left[n_{D_{1}}(n)\right.$, $\left.\ldots, n_{D_{K^{\prime}}}(n)\right]^{T}$.

Comparing (8) with (3), there are some differences listed as follows. (a) The elements of $\mathbf{h}_{S R_{k}}(n)$ in (3) are i.i.d because they represent the channel responses of different antennas on the same relay, while the elements of $\mathbf{h}_{R D}(n)$ in (8) are independent but not necessarily identically distributed (i.n.i.d). (b) The transmitting power $P_{S}$ from the source to each antenna of the $k$ th relay is equal, but the transmitting power $P_{R_{k}}$ from the $k$ th relay to the destination is not necessary to be equal. Due to these practical limitations, the analysis is somewhat different. In the following section, we will discuss them in detail.

At the destination, $\mathbf{R}_{n I_{D}}(n)$ is given by

$$
\mathbf{R}_{n I_{D}}(n)=P_{I} \sum_{m=1}^{N_{I}} \mathbf{h}_{I_{m} D}(n) \mathbf{h}_{I_{m} D}^{H}(n)+\sigma^{2} \mathbf{I}_{K^{\prime}}
$$

With the OC receiver, the maximum instantaneous SINR at the destination combiner output is similar to (5), which is given by

$$
\gamma_{D}(n)=\mathbf{P}_{R} \mathbf{h}_{R D}^{H}(n) \mathbf{R}_{n I_{D}}^{-1}(n) \mathbf{h}_{R D}(n)=\sum_{k=1}^{K^{\prime}} \frac{\sigma^{2}}{\lambda_{D_{k}}(n)} \gamma_{D_{k}}(n),
$$

where $\gamma_{D_{k}} \triangleq\left|h_{R_{k} D}(n)\right|^{2} P_{R_{k}} / \sigma^{2}, \lambda_{D_{1}}(n), \ldots, \lambda_{D_{K^{\prime}}}(n)$ are the eigenvalues of $\mathbf{R}_{n I_{D}}(n)$.

Since $\hat{h}_{x}(p), p=0, \ldots, L_{\text {tap }}-1, x \in\left\{S R_{k, l}, I_{m} R_{k, l}, R_{k} D\right.$, $\left.I_{m} D_{k}, l=0, \ldots, L-1, m=1, \ldots, N_{I}, k=1, \ldots, K\right\}$ are mutually independent, all the subcarriers $h_{x}(n), n=$ $0, \ldots, N_{\text {sub }}-1$ have the same distributions [23]. Hence, we assume in this paper that $h_{S R_{k, l}}(n) \in C N\left(0, \sigma_{S R_{k}}^{2}\right), h_{R_{k} D}(n) \in$ $C N\left(0, \sigma_{R_{k} D}^{2}\right), h_{I_{m} R_{k, l}}(n) \in C N\left(0, \sigma_{I_{m} R_{k}}^{2}\right)$, and $h_{I_{m} D_{k}}(n) \in$ $C N\left(0, \sigma_{I_{m} D}^{2}\right)$, where $\sigma_{S R_{k}}^{2}=\sum_{l=0}^{L_{\text {tap }}-1} \sigma_{S R_{k}, l}^{2}=\left(d_{S R_{k}}\right)^{-\mu}, \sigma_{R_{k} D}^{2}=$ $\sum_{l=0}^{L_{\mathrm{tap}}-1} \sigma_{R_{k} D, l}^{2}=\left(d_{R_{k} D}\right)^{-\mu}, \sigma_{I_{m} R_{k}}^{2}=\sum_{l=0}^{L_{\mathrm{tap}}-1} \sigma_{I_{m} R_{k}, l}^{2}=\left(d_{I_{m} R_{k}}\right)^{-\mu}$, $\sigma_{I_{m} D}^{2}=\sum_{l=0}^{L_{\text {tap }}-1} \sigma_{I_{m} D, l}^{2}=\left(d_{I_{m} D}\right)^{-\mu}, d_{A B}$ denotes the distance between node $\mathrm{A}$ and node $\mathrm{B}$, and $\mu$ is the path loss exponent. To be convenient for analysis, we assume $N_{I}$ interferers are all located nearly (e.g., at the edge of the cell), so the distance between each of them to any node (relay or destination) can be considered to be approximately equal, that is, $\sigma_{I_{1} D}^{2} \doteq$ $\cdots \doteq \sigma_{I_{N_{I}} D}^{2} \triangleq \sigma_{I D}^{2}=\left(d_{I_{1} D}\right)^{-\mu}$ and $\sigma_{I_{1} R_{k}}^{2}=\sigma_{I_{2} R_{k}}^{2}=\cdots=$ $\sigma_{I_{N_{I}} R_{k}}^{2} \triangleq \sigma_{I R_{k}}^{2}=\left(d_{I_{1} R_{k}}\right)^{-\mu}$.

\section{Performance Analysis}

As mentioned above, these subcarriers are orthogonal to each other. For the sake of simplicity, we assume that all subcarriers experience independent flat Rayleigh fading similar to [24]. Without loss of generality, the analysis will be aimed at the $n$th subcarrier.

Suppose that in the second phase $K^{\prime}\left(K^{\prime} \in[0, K]\right)$ relays, which can properly decode the received signal, would be selected to retransmit to the destination node, and the other $K-K^{\prime}$ relays will keep silence. Utilizing the total probability formula, the average SER of the $n$th subcarrier at the destination node with $M$-PSK modulation can be expressed as

$$
\begin{aligned}
P_{e}(n)= & \sum_{K^{\prime}=0}^{K} P_{n}\left(e \mid K^{\prime} \text { relays are right }\right) \\
& \cdot P_{n}\left(K^{\prime} \text { relays are right }\right),
\end{aligned}
$$

where $P_{n}\left(e \mid K^{\prime}\right.$ relays are right $)$ is the conditional SER of the $n$th subcarrier at the destination, conditioned on the situation that only $K^{\prime}$ relays can decode the signal properly on the $n$th subcarrier. $P_{n}\left(K^{\prime}\right.$ relays are right $)$ represents the probability of only $K^{\prime}$ relays decoding the signal properly on the $n$th subcarrier. In what follows, we will discuss the two phases, respectively.

3.1. The First Phase. In order to obtain $P_{n}\left(K^{\prime}\right.$ relays are right), let us consider the SER of the $n$th subcarrier at the $k$ th relay. Similar to [16], the interference correlation matrix $\mathbf{R}_{I_{k}}(n)$ is defined as

$$
\mathbf{R}_{I_{k}}(n)=\sum_{m=1}^{N_{I}} \mathbf{h}_{I_{m} R_{k}}(n) \mathbf{h}_{I_{m} R_{k}}^{H}(n)
$$

In [16], the authors suppose that all the channel responses are i.i.d. zero-mean unit variance complex Gaussian random variables and obtain the probability density function (pdf) of eigenvalues of the interference correlation 
matrix [16, Eq.6, Eq.8]. In order to make full use of these facts, we can rewrite (4) and (12) as follows:

$$
\begin{gathered}
\mathbf{R}_{n I_{k}}(n)=P_{I} \sigma_{I R_{k}}^{2} \sum_{m=1}^{N_{I}} \tilde{\mathbf{h}}_{I_{m} R_{k}}(n) \tilde{\mathbf{h}}_{I_{m} R_{k}}^{H}(n)+\sigma^{2} \mathbf{I}_{L} \\
\widetilde{\mathbf{R}}_{I_{k}}(n)=\sum_{m=1}^{N_{I}} \tilde{\mathbf{h}}_{I_{m} R_{k}}(n) \tilde{\mathbf{h}}_{I_{m} R_{k}}^{H}(n),
\end{gathered}
$$

where $\tilde{\mathbf{h}}_{I_{m} R_{k}}(n)=\mathbf{h}_{I_{m} R_{k}}(n) /\left|\mathbf{h}_{I_{m} R_{k}}(n)\right|, \tilde{\mathbf{h}}_{I_{m} R_{k}}(n) \in C N\left(0, \mathbf{I}_{L}\right)$. Let $N_{\min } \triangleq \min \left\{L, N_{I}\right\}, N_{\max } \triangleq \max \left\{L, N_{I}\right\}$, and $A_{k}(n)$ be the $N_{\min } \times N_{\min }$ Vandermonde matrix [25], whose determinant is

$$
\begin{aligned}
\left|A_{k}(n)\right|=\left|\begin{array}{cccc}
1 & 1 & \cdots & 1 \\
\lambda_{I_{k}, 1}(n) & \lambda_{I_{k}, 2}(n) & \cdots & \lambda_{I_{k}, N_{\min }}(n) \\
\vdots & \vdots & \cdots & \vdots \\
\lambda_{I_{k}, 1}^{N_{\min }-1}(n) & \lambda_{I_{k}, 2}^{N_{\min }-1}(n) & \cdots & \lambda_{I_{k}, N_{\min }}^{N_{\min }-1}(n)
\end{array}\right| \\
=\prod_{1 \leq i<j \leq N_{\min }}\left(\lambda_{I_{k}, j}(n)-\lambda_{I_{k}, i}(n)\right) .
\end{aligned}
$$

Then, the joint pdf of eigenvalues $\lambda_{I_{k}, 1}(n), \ldots, \lambda_{I_{k}, N_{\min }}(n)$ of $\widetilde{\mathbf{R}}_{I_{k}}(n)$ can be written as

$$
\begin{aligned}
& p_{I_{I_{k}, 1}(n), \ldots, \lambda_{I_{k}, N_{\min }}(n)}\left(\lambda_{I_{k}, 1}(n), \ldots, \lambda_{I_{k}, N_{\min }}(n)\right) \\
& =\frac{\left[\prod_{l=1}^{N_{\min }} \lambda_{I_{k}, l}(n)\right]^{N_{\max }-N_{\min }} \exp \left(-\sum_{l=1}^{N_{\min }} \lambda_{I_{k}, l}(n)\right)}{N_{\min } !\left[\prod_{l=1}^{N_{\min }}\left(N_{\min }-l\right) !\left(N_{\max }-l\right) !\right]}\left|A_{k}(n)\right|^{2},
\end{aligned}
$$

where $\lambda_{I_{k}, 1}(n), \ldots, \lambda_{I_{k}, N_{\text {min }}}(n)>0$. It is clear from (14) that the rank of $\widetilde{\mathbf{R}}_{I_{k}}(n)$ is $\min \left\{L, N_{I}\right\}$ when $N_{I} \geq L, \widetilde{\mathbf{R}}_{I_{k}}(n)$ is full rank $L$ and has $L$ eigenvalues, but when $N_{I}<L, \widetilde{\mathbf{R}}_{I_{k}}(n)$ is rank-deficient and its $L-N_{I}$ eigenvalues are zero.

For $M$-PSK modulation in the presence of Rayleigh fading, the conditional SER at the $k$ th relay can be written as [21]

$$
\begin{aligned}
P_{k, n} & \left(e \mid \lambda_{I_{k}, 1}(n), \ldots, \lambda_{I_{k}, N_{\min }}(n)\right) \\
\quad= & \frac{1}{\pi} \int_{0}^{(M-1) \pi / M} M_{\gamma_{k}(n) \mid \lambda_{I_{k}, 1}(n), \ldots, \lambda_{I_{k}, N_{\min }}(n)}\left(-\frac{g_{\mathrm{PSK}}}{\sin ^{2} \theta}\right) \mathrm{d} \theta,
\end{aligned}
$$

where $M_{\gamma_{k}(n) \mid \lambda_{I_{k}, 1}(n), \ldots, \lambda_{I_{k}, N_{\min }}(n)}$ is the conditional moment generating function (MGF) of $\gamma_{k}(n), g_{\mathrm{PSK}}=\sin ^{2} \pi / M$. From the foregoing analysis, $\lambda_{k, 1}(n), \ldots, \lambda_{k, N_{\min }}(n)$ and $\lambda_{I_{k}, 1}(n), \ldots, \lambda_{I_{k}, N_{\text {min }}}(n)$ are the nonzero eigenvalues of $\mathbf{R}_{n I_{k}}(n)$ and $\widetilde{\mathbf{R}}_{I_{k}}(n)$, respectively. From (13) and (14), it can be shown that $\mathbf{R}_{n I_{k}}(n)=\left(P_{I} / \sigma_{I R_{k}}^{2}\right) \widetilde{\mathbf{R}}_{I_{k}}(n)+\sigma^{2} \mathbf{I}_{L}$. Then, $\lambda_{k, j}(n)$ and $\lambda_{I_{k}, j}(n)$ can be related as

$$
\lambda_{k, j}(n)=P_{I} \sigma_{I R_{k}}^{2} \lambda_{I_{k}, j}(n)+\sigma^{2} \quad\left(j \in\left[1, N_{\min }\right]\right),
$$

Substituting (18) into (5), we obtain

$$
\gamma_{k}(n)=P_{S} \sum_{l=1}^{N_{\min }} \frac{\left|h_{S R_{k, l}}(n)\right|^{2}}{P_{I} \sigma_{I R_{k}}^{2} \lambda_{I_{k}, l}(n)+\sigma^{2}}+P_{S} \sum_{l=N_{\min }+1}^{L} \frac{\left|h_{S R_{k, l}}(n)\right|^{2}}{\sigma^{2}} .
$$

Since $\mathbf{h}_{S R_{k}}(n)$ has a $C N\left(0, \sigma_{S R_{k}}^{2} \mathbf{I}_{L}\right)$ distribution, $M_{\gamma_{k}(n) \mid \lambda_{I_{k}, 1}(n), \ldots, \lambda_{I_{k}, N_{\min }}(n)}$ can be expressed as [16]

$$
\begin{aligned}
M_{\gamma_{k}(n) \mid \lambda_{I_{k}, 1}(n), \ldots, \lambda_{I_{k}, N_{\min }}(n)}(s) & =\frac{1}{\left(1-s\left(P_{S} \sigma_{S R_{k}}^{2} / \sigma^{2}\right)\right)^{L-N_{\min }}} \\
& \times \frac{1}{\prod_{l=1}^{N_{\min }}\left(1-s\left(P_{S} \sigma_{S R_{k}}^{2} /\left(P_{I} \sigma_{I R_{k}}^{2} \lambda_{I_{k}, l}(n)+\sigma^{2}\right)\right)\right)} .
\end{aligned}
$$

Substituting (20) into (17), we obtain

$$
\begin{aligned}
& P_{k, n}\left(e \mid \lambda_{I_{k}, 1}(n), \ldots, \lambda_{I_{k}, N_{\min }}(n)\right) \\
& =\frac{1}{\pi} \int_{0}^{(M-1) \pi / M}\left(\frac{\sin ^{2} \theta}{\sin ^{2} \theta+\left(g_{\mathrm{PSK}} P_{S} \sigma_{S R_{k}}^{2} / \sigma^{2}\right)}\right)^{L-N_{\min }} \\
& \quad \times \prod_{l=1}^{N_{\min }}\left(\frac{\sin ^{2} \theta}{\sin ^{2} \theta+\left(g_{\mathrm{PSK}} P_{S} \sigma_{S R_{k}}^{2} /\left(P_{I} \sigma_{I R_{k}}^{2} \lambda_{I_{k}, l}(n)+\sigma^{2}\right)\right)}\right) d \theta .
\end{aligned}
$$

So the average SER of the $n$th subcarrier at the $k$ th relay can be expressed as

$$
\begin{aligned}
P_{k, n}(e)= & \int_{0}^{\infty} \cdots \int_{0}^{\infty} P_{k, n}\left(e \mid \lambda_{I_{k}, 1}(n), \ldots, \lambda_{I_{k}, N_{\min }}(n)\right) \\
& \times p_{\lambda_{I_{k}, 1}(n), \ldots, \lambda_{I_{k}, N_{\min }}(n)}\left(\lambda_{I_{k}, 1}(n), \ldots, \lambda_{I_{k}, N_{\min }}(n)\right) \\
& \times d \lambda_{I_{k}, 1}(n), \ldots, \lambda_{I_{k}, N_{\min }}(n)
\end{aligned}
$$

Equation (23) is the SER for M-PSK with OC at the $k$ th relay in the presence of $N_{I}$ interferers. But it requires to evaluate an $N_{\text {min }}$-fold integral. In [16], by utilizing averaging techniques to exploit the properties of the Vandermonde determinant, the authors obtain the closed-form BER expressions for $N_{I}<L$ and $N_{I} \geq L$, respectively. But the results are only suitable for BPSK modulation under the assumption that all the channel responses are i.i.d zero-mean unit variance complex Gaussian random variables, which can be considered as a special case in our paper. 
3.1.1. $N_{I} \geq L$. Let $N_{\min }=L, N_{\max }=N_{I} \cdot P_{k, n}(e$ $\left.\lambda_{I_{k}, 1}(n), \ldots, \lambda_{I_{k}, N_{\min }}(n)\right)$ in (21), be rewritten using partial fraction expansion, that is

$$
\begin{aligned}
& P_{k, n}\left(e \mid \lambda_{I_{k}, 1}(n), \ldots, \lambda_{I_{k}, N_{\min }}(n)\right) \\
& =\frac{1}{\pi} \int_{0}^{(M-1) \pi / M} \prod_{l=1}^{N_{\min }} \\
& \times\left(\frac{\sin ^{2} \theta}{\sin ^{2} \theta+\left(g_{\mathrm{PSK}} P_{S} \sigma_{S R_{k}}^{2} /\left(P_{I} \sigma_{I R_{k}}^{2} \lambda_{I_{k}, l}(n)+\sigma^{2}\right)\right)}\right) d \theta \\
& =\sum_{l=1}^{L}\left[\prod_{\substack{j=1 \\
j \neq l}}^{L} \frac{P_{I} \sigma_{I R_{k}}^{2} \lambda_{I_{k}, j}(n)+\sigma^{2}}{P_{I} \sigma_{I R_{k}}^{2}\left(\lambda_{I_{k}, j}(n)-\lambda_{I_{k}, l}(n)\right)}\right] \\
& \times\left[\frac{1}{\pi} \int_{0}^{(M-1) \pi / M}\right. \\
& \left.\times\left(\frac{\sin ^{2} \theta}{\sin ^{2} \theta+\left(g_{\mathrm{PSK}} P_{S} \sigma_{S R_{k}}^{2} /\left(P_{I} \sigma_{I R_{k}}^{2} \lambda_{I_{k}, l}(n)+\sigma^{2}\right)\right)}\right) d \theta\right] .
\end{aligned}
$$

Using the [21, 5A.15], (23) can be rewritten as

$$
\begin{aligned}
& P_{k, n}\left(e \mid \lambda_{I_{k}, 1}(n), \ldots, \lambda_{I_{k}, N_{\min }}(n)\right) \\
& =\frac{M-1}{M} \sum_{l=1}^{L}\left[\prod_{j=1}^{L} \frac{P_{I} \sigma_{I R_{k}}^{2} \lambda_{I_{k}, j}(n)+\sigma^{2}}{P_{I} \sigma_{I R_{k}}^{2}\left(\lambda_{I_{k}, j}(n)-\lambda_{I_{k}, l}(n)\right)}\right] \\
& \quad \times\left[1-\sqrt{\frac{c}{1+c}} \frac{M}{(M-1) \pi}\left[\frac{\pi}{2}+\arctan \left(\sqrt{\frac{c}{1+c}} \cot \frac{\pi}{M}\right)\right],\right.
\end{aligned}
$$

where $c=g_{\mathrm{PSK}} P_{S} \sigma_{S R_{k}}^{2} /\left(P_{I} \sigma_{I R_{k}}^{2} \lambda_{I_{k}, l}(n)+\sigma^{2}\right)$.

Following the same line in [16], we can obtain that

$$
\begin{aligned}
& P_{k, n}(e) \\
& =\frac{M-1}{M} \frac{1}{\prod_{l=1}^{L}(L-l) !\left(N_{I}-l\right) !} \\
& \quad \times \sum_{p=0}^{L-1} \beta_{p}\left[\int_{0}^{\infty} \lambda_{I_{k}}^{N_{I}-L+p}(n) \exp \left(-\lambda_{I_{k}}(n)\right) d \lambda_{I_{k}}(n)\right. \\
& \quad-\frac{M}{2(M-1)} \sqrt{\frac{g_{\mathrm{PSK}} P_{S} \sigma_{S R_{k}}^{2}}{P_{I} \sigma_{I R_{k}}^{2}}}
\end{aligned}
$$

$$
\begin{aligned}
& \times \int_{0}^{\infty} \frac{\lambda_{I_{k}}^{N_{I}-L+p}(n) \exp \left(-\lambda_{I_{k}}(n)\right)}{\sqrt{\lambda_{I_{k}}(n)+\left(\left(g_{\mathrm{PSK}} P_{S} \sigma_{S R_{k}}^{2}+\sigma^{2}\right) / P_{I} \sigma_{I R_{k}}^{2}\right)}} d \lambda_{I_{k}}(n) \\
& -\frac{M}{(M-1) \pi} \sqrt{\frac{g_{\mathrm{PSK}} P_{S} \sigma_{S R_{k}}^{2}}{P_{I} \sigma_{I R_{k}}^{2}}} \\
& \times \int_{0}^{\infty} \frac{\lambda_{I_{k}}^{N_{I}-L+p}(n) \exp \left(-\lambda_{I_{k}}(n)\right)}{\sqrt{\lambda_{I_{k}}(n)+\left(\left(g_{\mathrm{PSK}} P_{S} \sigma_{S R_{k}}^{2}+\sigma^{2}\right) / P_{I} \sigma_{I R_{k}}^{2}\right)}} \\
& \times \arctan \left(\frac{\sqrt{g_{\mathrm{PSK}} P_{S} \sigma_{S R_{k}}^{2} / P_{I} \sigma_{I R_{k}}^{2}} \cot (\pi / M)}{\sqrt{\lambda_{I_{k}}(n)+\left(\left(g_{\mathrm{PSK}} P_{S} \sigma_{S R_{k}}^{2}+\sigma^{2}\right) / P_{I} \sigma_{I R_{k}}^{2}\right)}}\right) \\
& \left.\times d \lambda_{I_{k}}(n)\right],
\end{aligned}
$$

where,

$$
\begin{aligned}
\beta_{p}= & {\left[\prod_{l=1}^{L}(L-1-l) !\left(N_{I}-1-l\right) !\right] } \\
& \times(-1)^{p}\left(\begin{array}{c}
L-1 \\
p
\end{array}\right) \frac{\left(N_{I}-1\right) !}{\left(N_{I}-L+p\right) !} \\
& \times \sum_{l=0}^{L-1} \alpha_{p, l}\left(\frac{\sigma^{2}}{P_{I} \sigma_{I R_{k}}^{2}}\right)^{l}, \\
\alpha_{p, l}= & \min (p, L-1-l) \\
& \times \frac{(-1)^{i}\left(\begin{array}{c}
p \\
i
\end{array}\right)\left(\begin{array}{c}
L-1-i \\
l
\end{array}\right)}{\left(N_{I}-L+l+i+1\right) !} .
\end{aligned}
$$

The function $g_{m}(a)$ is defined in [16] as

$$
\begin{aligned}
g_{m}(a)= & \int_{0}^{\infty} \frac{\lambda_{I_{k}}^{m}(n) \exp \left(-\lambda_{I_{k}}(n)\right)}{\sqrt{\lambda_{I_{k}}(n)+a}} d \lambda_{I_{k}}(n) \\
= & \sum_{i=0}^{m} \Gamma(i+0.5)\left(\begin{array}{c}
m \\
i
\end{array}\right)(-a)^{m-i} \\
& \times\left[2 \exp (a) Q(\sqrt{2 a})+\sum_{j=1}^{i} \frac{a^{j-0.5}}{\Gamma(j+0.5)}\right] .
\end{aligned}
$$

In this paper, we introduce the functions $\varphi_{m}(a, b)$ as

$$
\begin{aligned}
\varphi_{m}(a, b)= & \int_{0}^{\infty} \frac{\lambda_{I_{k}}^{m}(n) \exp \left(-\lambda_{I_{k}}(n)\right)}{\sqrt{\lambda_{I_{k}}(n)+a}} \\
& \times \arctan \left(\frac{b}{\sqrt{\lambda_{I_{k}}(n)+a}}\right) d \lambda_{I_{k}}(n), \\
& a>0, \quad m=0,1,2, \ldots
\end{aligned}
$$


Its closed-form expression is given by (A.11) of Appendix A. Thus, (25) can be rewritten as

$$
\begin{aligned}
& P_{k, n}(e) \\
& =\frac{M-1}{M} \frac{1}{\prod_{l=1}^{L}(L-l) !\left(N_{I}-l\right) !} \\
& \times \sum_{p=0}^{L-1} \beta_{p}\left[\left(N_{I}-L+p\right) !-\frac{M}{2(M-1)}\right. \\
& \quad \times \sqrt{\frac{g_{\mathrm{PSK}} P_{S} \sigma_{S R_{k}}^{2}}{P_{I} \sigma_{I R_{k}}^{2}} g_{N_{I}-L+p}\left(\frac{g_{\mathrm{PSK}} P_{S} \sigma_{S R_{k}}^{2}+\sigma^{2}}{P_{I} \sigma_{I R_{k}}^{2}}\right)} \\
& \quad-\frac{M}{(M-1) \pi} \sqrt{\frac{g_{\mathrm{PSK}} P_{S} \sigma_{S R_{k}}^{2}}{P_{I} \sigma_{I R_{k}}^{2}}} \\
& \left.\quad \times \phi_{N_{I}-L+p}\left(\frac{g_{\mathrm{PSK}} P_{S} \sigma_{S R_{k}}^{2}+\sigma^{2}}{P_{I} \sigma_{I R_{k}}^{2}}, \sqrt{\frac{g_{\mathrm{PSK}} P_{S} \sigma_{S R_{k}}^{2}}{P_{I} \sigma_{I R_{k}}^{2}}} \cot \frac{\pi}{M}\right)\right]
\end{aligned}
$$

which is the closed-form SER expression of the $n$th subcarrier at the $k$ th relay when $N_{I} \geq L$.

3.1.2. $N_{I}<L$. Let $N_{\min }=N_{I}, N_{\max }=L . P_{k, n}(e$ | $\left.\lambda_{I_{k}, 1}(n), \ldots, \lambda_{I_{k}, N_{\min }}(n)\right)$ in (21) be rewritten using partial fraction expansion, that is,

$$
\begin{aligned}
& P_{k, n}\left(e \mid \lambda_{I_{k}, 1}(n), \ldots, \lambda_{I_{k}, N_{\min }}(n)\right) \\
& =\frac{1}{\pi} \int_{0}^{(M-1) \pi / M}\left(\frac{\sin ^{2} \theta}{\sin ^{2} \theta+\left(g_{\mathrm{PSK}} P_{S} \sigma_{S R_{k}}^{2} / \sigma^{2}\right)}\right)^{L-N_{I}} \\
& \times \prod_{l=1}^{N_{I}}\left(\frac{\sin ^{2} \theta}{\sin ^{2} \theta+\left(g_{\mathrm{PSK}} P_{S} \sigma_{S R_{k}}^{2} /\left(P_{I} \sigma_{I R_{k}}^{2} \lambda_{I_{k}, l}(n)+\sigma^{2}\right)\right)}\right) d \theta \\
& =\sum_{l=1}^{N_{I}}\left[\prod_{\substack{j=1 \\
j \neq l}}^{N_{I}} \frac{P_{I} \sigma_{I R_{k}}^{2} \lambda_{I_{k}, j}(n)+\sigma^{2}}{P_{I} \sigma_{I R_{k}}^{2}\left(\lambda_{I, j}(n)-\lambda_{I_{k}, l}(n)\right)}\right] \\
& \times\left[\frac{1}{\pi} \int_{0}^{(M-1) \pi / M}\left(\frac{\sin ^{2} \theta}{\sin ^{2} \theta+\left(g_{\mathrm{PSK}} P_{S} \sigma_{S R_{k}}^{2} / \sigma^{2}\right)}\right)^{L-N_{I}}\right. \\
& \left.\times\left(\frac{\sin ^{2} \theta}{\sin ^{2} \theta+\left(g_{\mathrm{PSK}} P_{S} \sigma_{S R_{k}}^{2} /\left(P_{I} \sigma_{I R_{k}}^{2} \lambda_{I_{k}, l}(n)+\sigma^{2}\right)\right)}\right) d \theta\right] .
\end{aligned}
$$

Hence, $P_{k, n}(e)$ can be written as (detailed derivations can be seen in Appendix B)

$$
\begin{aligned}
& P_{k, n}(e) \\
& =\frac{1}{\left[\prod_{l=1}^{N_{I}}\left(N_{I}-l\right) !(L-l) !\right]} \\
& \times \sum_{p=0}^{N_{I}-1} \beta_{p}\left\{I_{L-N_{I}}\left(\frac{(M-1) \pi}{M} ; c_{1}\right)\right. \\
& \times\left(L-N_{I}+p\right) !+\frac{T_{1}}{\pi} \sqrt{\frac{c_{1}}{1+c_{1}}} \sum_{q=0}^{L-N_{I}-1}\left(\begin{array}{c}
2 q \\
q
\end{array}\right) \\
& \times \frac{(q+p) !}{\left[4\left(1+c_{1}\right)\right]^{q}}\left(-\frac{\sigma^{2}}{P_{I} \sigma_{I R_{k}}^{2}}\right)^{L-N_{I}-q} \\
& +\frac{2}{\pi} \sqrt{\frac{c_{1}}{1+c_{1}}} \sum_{q=0}^{L-N_{I}-1} \sum_{t=0}^{q-1}\left(\begin{array}{c}
2 q \\
t
\end{array}\right) \\
& \times \frac{(-1)^{t+q}(q+p) !}{\left[4\left(1+c_{1}\right)\right]^{q}} \frac{\sin \left[(2 q-2 t) T_{1}\right]}{2 q-2 t} \\
& \times\left(-\frac{\sigma^{2}}{P_{I} \sigma_{I R_{k}}^{2}}\right)^{L-N_{I}-q} \\
& -\int_{0}^{\infty} \lambda_{I_{k}, l}^{p}(n) \exp \left(-\lambda_{I_{k}, l}(n)\right) \frac{T_{2}}{\pi} \sqrt{\frac{g_{\mathrm{PSK}} P_{S} \sigma_{S R_{k}}^{2}}{P_{I} \sigma_{I R_{k}}^{2}}} \\
& \times \sqrt{\frac{1}{\lambda_{I_{k}, l}(n)+\left(\left(g_{\mathrm{PSK}} P_{S} \sigma_{S R_{k}}^{2}+\sigma^{2}\right) / P_{I} \sigma_{I R_{k}}^{2}\right)}} \\
& \left.\times\left(-\frac{\sigma^{2}}{P_{I} \sigma_{I R_{k}}^{2}}\right)^{L-N_{I}} d \lambda_{I_{k}, l}(n)\right\} .
\end{aligned}
$$

Now, we focus on the evaluation of the integral $B$ defined as

$$
\begin{aligned}
B= & -\int_{0}^{\infty} \lambda_{I_{k}, l}^{p}(n) \exp \left(-\lambda_{I, l}, l(n)\right) \frac{T_{2}}{\pi} \sqrt{\frac{g_{\mathrm{PSK}} P_{S} \sigma_{S R_{k}}^{2}}{P_{I} \sigma_{I R_{k}}^{2}}} \\
& \times \sqrt{\frac{1}{\lambda_{I_{k}, l}(n)+\left(\left(g_{\mathrm{PSK}} P_{S} \sigma_{S R_{k}}^{2}+\sigma^{2}\right) / P_{I} \sigma_{I R_{k}}^{2}\right)}} \\
& \times\left(-\frac{\sigma^{2}}{P_{I} \sigma_{I R_{k}}^{2}}\right)^{L-N_{I}} d \lambda_{I_{k}, l}(n) .
\end{aligned}
$$

After some mathematic transformations, a closed-form expression of $B$ is given by (C.7) of Appendix C. Thus, substituting (C.7) into (31), we can obtain the closed-form expression for the SER of the $n$th subcarrier at the $k$ th relay 
when $N_{I}<L$ as follows:

$$
\begin{aligned}
& P_{k, n}(e) \\
& =\frac{1}{\left[\prod_{l=1}^{N_{I}}\left(N_{I}-l\right) !(L-l) !\right]} \\
& \times \sum_{p=0}^{N_{I}-1} \beta_{p}\left\{I_{L-N_{I}}\left(\frac{(M-1) \pi}{M} ; c_{1}\right)\left(L-N_{I}+p\right) !\right. \\
& +\frac{T_{1}}{\pi} \sqrt{\frac{c_{1}}{1+c_{1}}} \sum_{q=0}^{L-N_{I}-1}\left(\begin{array}{c}
2 q \\
q
\end{array}\right) \frac{(q+p) !}{\left[4\left(1+c_{1}\right)\right]^{q}} \\
& \times\left(-\frac{\sigma^{2}}{P_{I} \sigma_{I R_{k}}^{2}}\right)^{L-N_{I}-q}+\frac{2}{\pi} \sqrt{\frac{c_{1}}{1+c_{1}}} \\
& \times \sum_{q=0}^{L-N_{I}-1} \sum_{t=0}^{q-1}\left(\begin{array}{c}
2 q \\
t
\end{array}\right) \times \frac{(-1)^{t+q}(q+p) !}{\left[4\left(1+c_{1}\right)\right]^{q}} \\
& \times \frac{\sin \left[(2 q-2 t) T_{1}\right]}{2 q-2 t}\left(-\frac{\sigma^{2}}{P_{I} \sigma_{I R_{k}}^{2}}\right)^{L-N_{I}-q} \\
& -\frac{1}{2}\left[\frac{1}{2} \operatorname{sgn}\left(\operatorname{sgn}\left(\frac{N_{2}}{D_{2}}\right)+\frac{1}{2}\right)+\frac{1}{2}\right. \\
& \left.-\operatorname{sgn}\left(N_{2}\right) \frac{1+\operatorname{sgn}\left(D_{2}\right)}{2}\right] \\
& \times \sqrt{\frac{g_{\mathrm{PSK}} P_{S} \sigma_{S R_{k}}^{2}}{P_{I} \sigma_{I R_{k}}^{2}}} g_{p}\left(\frac{g_{\mathrm{PSK}} P_{S} \sigma_{S R_{k}}^{2}+\sigma^{2}}{P_{I} \sigma_{I R_{k}}^{2}}\right) \\
& \times\left(-\frac{\sigma^{2}}{P_{I} \sigma_{I R_{k}}^{2}}\right)^{L-N_{I}}-\frac{1}{2 \pi} \sqrt{\frac{g_{\mathrm{PSK}} P_{S} \sigma_{S R_{k}}^{2}}{P_{I} \sigma_{I R_{k}}^{2}}} \\
& \times\left(-\frac{\sigma^{2}}{P_{I} \sigma_{I R_{k}}^{2}}\right)^{L-N_{I}} \\
& \times \varphi_{p}\left(\frac{g_{\mathrm{PSK}} P_{S} \sigma_{S R_{k}}^{2}+\sigma^{2}}{P_{I} \sigma_{I R_{k}}^{2}}, \frac{\sin 2 \phi}{\cos 2 \phi-1} \sqrt{\frac{g_{\mathrm{PSK}} P_{S} \sigma_{S R_{k}}^{2}}{P_{I} \sigma_{I R_{k}}^{2}}}\right) \\
& +\frac{1}{2 \pi} \sqrt{\frac{g_{\mathrm{PSK}} P_{S} \sigma_{S R_{k}}^{2}}{P_{I} \sigma_{I R_{k}}^{2}}}\left(-\frac{\sigma^{2}}{P_{I} \sigma_{I R_{k}}^{2}}\right)^{L-N_{I}} \\
& \left.\times \varphi_{p}\left(\frac{g_{\mathrm{PSK}} P_{S} \sigma_{S R_{k}}^{2}+\sigma^{2}}{P_{I} \sigma_{I R_{k}}^{2}}, \frac{\cos 2 \phi+1}{\sin 2 \phi} \sqrt{\frac{g_{\mathrm{PSK}} P_{S} \sigma_{S R_{k}}^{2}}{P_{I} \sigma_{I R_{k}}^{2}}}\right)\right\} .
\end{aligned}
$$

Combining (29) and (33), we obtain the closed-form SER expression of the $n$th subcarrier at the $k$ th relay. Thus, $P_{n}\left(K^{\prime}\right.$ relays are right) in (11) can be expressed as

$$
P_{n}\left(K^{\prime} \text { relays are right }\right)
$$

$$
=\sum_{k_{1}+k_{2}+\cdots+k_{K}=K^{\prime}, k_{i} \in\{0,1\}} \prod_{m=1}^{K} P_{m, n}^{1-k_{m}}(e)\left(1-P_{m, n}(e)\right)^{k_{m}},
$$

where $k_{i}(i \in[1, K])$ represents whether the decoded symbol of the $n$th subcarrier at the $i$ th relay is right or not; that is, $k_{i}=1$ represents right decoding and vice versa.

3.2. The Second Phase. As mentioned before, there are some important differences between these two phases, as just did in Section 3.1, $\mathbf{R}_{n I_{D}}(n)$ and $\widetilde{\mathbf{R}}_{I_{D}}(n)$ in (9) can be rewritten as

$$
\begin{gathered}
\mathbf{R}_{n I_{D}}(n)=P_{I} \sigma_{I D}^{2} \sum_{m=1}^{N_{I}} \tilde{\mathbf{h}}_{I_{m} D}(n) \tilde{\mathbf{h}}_{I_{m} D}^{H}(n)+\sigma^{2} \mathbf{I}_{K^{\prime}}, \\
\widetilde{\mathbf{R}}_{I_{D}}(n)=\sum_{m=1}^{N_{I}} \tilde{\mathbf{h}}_{I_{m} D}(n) \tilde{\mathbf{h}}_{I_{m} D}^{H}(n),
\end{gathered}
$$

where $\widetilde{\mathbf{h}}_{I_{m} D}(n)=\mathbf{h}_{I_{m} D}(n) /\left|\mathbf{h}_{I_{m} D}(n)\right|, \widetilde{\mathbf{h}}_{I_{m} D}(n) \in C N\left(0, \mathbf{I}_{K^{\prime}}\right)$.

Let $\lambda_{I_{D}, 1}(n), \ldots, \lambda_{I_{D}, N_{\min }}(n)$ be the nonzero eigenvalues of $\widetilde{\mathbf{R}}_{I_{D}}(n)$, so $M_{\gamma_{D}(n) \mid \lambda_{I_{D}, 1}(n), \ldots, \lambda_{I_{D}, N_{\text {min }}}(n)}(s)$ can be expressed as [16]

$$
\begin{aligned}
M_{\gamma_{D}(n) \mid \lambda_{I_{D}, 1}(n), \ldots, \lambda_{I_{D}, N_{\min }}(n)}(s) & \\
= & \frac{1}{\prod_{k=N_{\min }+1}^{K^{\prime}}\left(1-s\left(P_{R_{k}} \sigma_{R_{k} D}^{2} / \sigma^{2}\right)\right)} \\
& \times \frac{1}{\prod_{k=1}^{N_{\min }}\left(1-s\left(P_{R_{k}} \sigma_{R_{k} D}^{2} /\left(P_{I} \sigma_{I D}^{2} \lambda_{I_{D}, k}(n)+\sigma^{2}\right)\right)\right)} .
\end{aligned}
$$

For convenience, we suppose each relay has its corresponding transmitting power $P_{R_{k}}=P_{S} / \sigma_{R_{k} D}^{2}$, and (36) can be rewritten as

$$
\begin{aligned}
M_{\gamma_{D}(n) \mid \lambda_{I_{D}, 1}(n), \ldots, \lambda_{I_{D}, N_{\min }}(n)}(s) & =\frac{1}{\left(1-s\left(P_{S} / \sigma^{2}\right)\right)^{K^{\prime}-N_{\min }}} \\
& \times \frac{1}{\prod_{k=1}^{N_{\min }}\left(1-s\left(P_{S} /\left(P_{I} \sigma_{I D}^{2} \lambda_{I_{D}, k}(n)+\sigma^{2}\right)\right)\right)} .
\end{aligned}
$$

Comparing (37) with (20), it can be found that the analysis for the second phase can be simplified to the situation with i.i.d channel responses because of utilizing this kind of simple power allocation. Therefore, $P_{n}(e \quad \mid$ $K^{\prime}$ relays are right) can be calculated by the same analytical method which is used at the $k$ th relay node. By using $K^{\prime}$ instead of $L, \sigma_{I D}^{2}$, instead of $\sigma_{I R_{k}}^{2}$ and $\sigma_{S R_{k}}^{2}=1$, the closed-form expression for $P_{n}\left(e \mid K^{\prime}\right.$ relays are right) when $N_{I} \geq K^{\prime}$ and $N_{I}<K^{\prime}$ is given by (29) and (33), respectively.

Now, we can obtain the average SER of the $n$th subcarrier at the destination with $M$-PSK modulation as follows:

$$
\begin{aligned}
P_{e}(n)= & \sum_{K^{\prime}=0}^{K} P_{n}\left(e \mid K^{\prime} \text { relays are right }\right) \\
& \cdot \sum_{k_{1}+k_{2}+\cdots+k_{K}=K^{\prime}, k_{i} \in\{0,1\}} \prod_{m=1}^{K} P_{m, n}^{1-k_{m}}(e)\left(1-P_{m, n}(e)\right)^{k_{m}} .
\end{aligned}
$$

Equation (38) is the closed-form SER expression of the $n$th subcarrier for DF relays in the presence of $N_{I}$ interferers. 
Thus, the average SER of the whole system is

$$
P_{\text {SER }}=\frac{1}{N_{\text {sub }}} \sum_{n=1}^{N_{\text {sub }}} P_{e}(n)
$$

Since all the subcarriers of one OFDM symbol have the same distributions [23], $P_{e}(n)$ of all subcarriers at the destination are equal. It means that

$$
P_{\mathrm{SER}}=P_{e}(n) \text {. }
$$

When a Gray code is used in the mapping, the equivalent bit error probability for M-PSK is well approximated as [26]

$$
P_{b} \approx \frac{1}{\log _{2} M} P_{\text {SER }}
$$

As mentioned above, because of OFDM technology, the frequency-selective fading channel is converted to $N_{\text {sub }}$ flat-fading channels, and all subcarriers are assumed to experience independent flat Rayleigh fading similar to [24]. Thus, no frequency diversity can be observed in this system, and the diversity order of the whole system is equal to that of the $n$th subcarrier.

According to [27], the diversity order $d$ of the $n$th subcarrier is defined as

$$
d=-\lim _{\mathrm{SNR} \rightarrow \infty} \frac{\log P_{e}(n)}{\log \mathrm{SNR}} .
$$

Intuitively, the definition can be understood as following. The term $\log$ SNR is the capacity of a single-antenna channel. Spatial diversity $d$ is the SNR exponent of the error probability and corresponds to the number of independently faded paths that a symbol passes through. In this paper, because we use multiple DF relays with SNR threshold, the selected relays retransmit the same signals to the destination node in the TDM mode. It can be well understood that this cooperative transmission is similar to the single-inputmultiple-output (SIMO) channel. The diversity order of traditional SIMO channel is discussed in many papers, such as $[16,21,28]$. So, in this paper, the diversity order of the $n$th subcarrier can be considered to be equal to the number of the selected relays $K^{\prime}$, where $K^{\prime} \in[0, K]$. If $P_{S} \gg P_{I}$, we can ignore the effects of the interferers and get the diversity order $d=K$. However, if $P_{I}$ is comparable with $P_{S}$, the situation becomes complicated, we discuss it in following two aspects.

Firstly, we consider that $N_{I}<L$. When SNR $\rightarrow$ $\infty$, the term $\sigma^{2} / P_{I} \sigma_{I R_{k}}^{2}$ in (33) can be approximated as $\sigma^{2} / P_{I} \sigma_{I R_{k}}^{2} \rightarrow 0$. So $P_{k, n}(e)$ in (33) is approximately to be zero. Substitute $P_{k, n}(e)$ into (38), we can get that $P_{e}(n) \doteq P_{n}(e$ | $K$ relays are right). That is to say, $K$ relays will be selected to retransmit the signals. Hence, the diversity order of the $n$th subcarrier is equal to $K$.

Secondly, we consider that $N_{I} \geq L$. Although it is difficult for us to calculate the asymptotic value of $P_{k, n}(e)$ in (29), we can find that $P_{k, n}(e)$ in (29) increases as the value of $N_{I}-L$ increases. That is to say, the number of the selected relays is smaller than $K$, and it becomes smaller and smaller as the value of $N_{I}-L$ increases. Hence, the diversity order of the $n$th subcarrier is $\widetilde{K} \in[0, K)$.

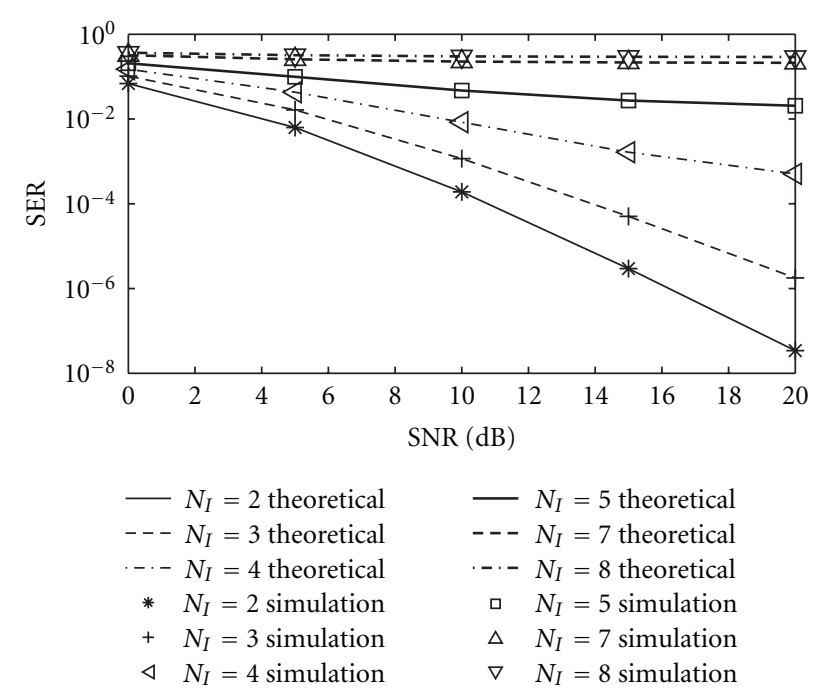

FIGURE 2: SER versus average SNR with different values of $N_{I}$ when $L=4, K=6$, and SIR $=0 \mathrm{~dB}$.

We conclude that the diversity order of the whole system is affected by the number of the interferers $N_{I}$, the number of the relay nodes $K$, and the number of the relay antennas $L$. In Section 4, we show how these parameters affect the diversity order through simulations.

\section{Numerical Results}

In this section, we verify our theoretical results through Monte-Carlo simulations. Simulations are carried out under the following settings: (1) the pathloss exponent is $\mu=$ 2 , (2) the SNR in the simulations is defined as $P_{s} / \sigma^{2}$ and $\sigma^{2}=1$, (3) it is assumed that one OFDM symbol has $N_{\text {sub }}=64$ subcarriers, and (4) we also assume that $L_{\text {tap }}=$ 6 and the power delay profile of each channel is uniform; that is, the tap of each channel is modeled as a zeromean Gaussian random variable with variance $\sigma_{x}^{2} / L_{\text {tap }}$, where $x \in\left\{S R_{k}, I_{m} R_{k}, R_{k} D, I_{m} D, m=1, \ldots, N_{I}, k=1, \ldots, K\right\} . \mathrm{A}$ pseudorandom frequency interleaver [29] is used, so after deinterleaving adjacent subcarriers become approximately uncorrelated.

For various values of $N_{I}, L$, and $K$, average signal-tointerference ratio (SIR) per branch $P_{s} / P_{I}$ and average SNR per branch $P_{s} / \sigma^{2}$, we compare the simulation values with our theoretical results, using QPSK modulation as one case of M-PSK. Meanwhile, we compare the SER performance of different modulation such as BPSK, QPSK, and 8PSK. In Figures 2-4 and Figure 6, we assume all interferers are generated as Gaussian distribution, but in Figure 5, both the desired signal and the interference signal are assumed to be QPSK. From these figures, we can find that the theoretical results match the simulation results well.

Figure 2 shows the SER performance of the considered system versus average SNR per branch $P_{s} / \sigma^{2}$ with different values of $N_{I}$, when QPSK modulation is used. In this figure, we consider $N_{I}=2,3,4,5,7,8, L=4$ and $K=6$ when SIR = $0 \mathrm{~dB}$. From Figure 2, it can be concluded that: (1) for a given 


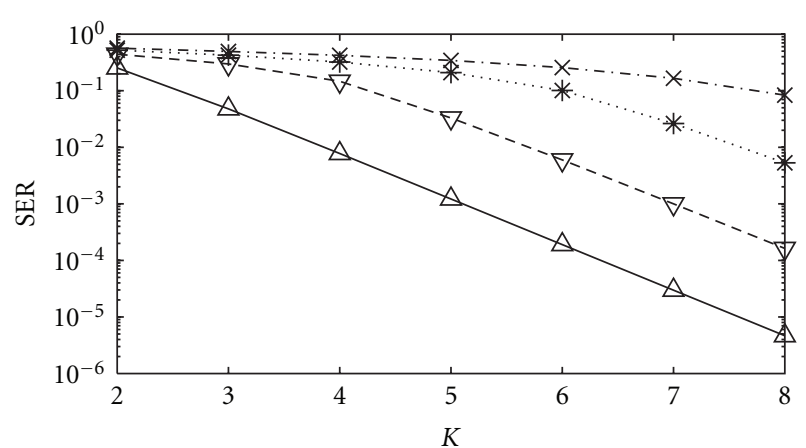

$\begin{array}{rlrl}- & N_{I}=2 \text { theoretical } & \triangle N_{I}=2 \text { simulation } \\ ---N_{I}=4 \text { theoretical } & \nabla N_{I}=4 \text { simulation } \\ \cdots \cdots N_{I}=6 \text { theoretical } & * N_{I}=6 \text { simulation } \\ \cdots--N_{I}=8 \text { theoretical } & \times N_{I}=8 \text { simulation }\end{array}$

FIgure 3: SER versus $K$ with different values of $N_{I}$ when $L=6$, SIR $=0 \mathrm{~dB}$, and SNR $=10 \mathrm{~dB}$.

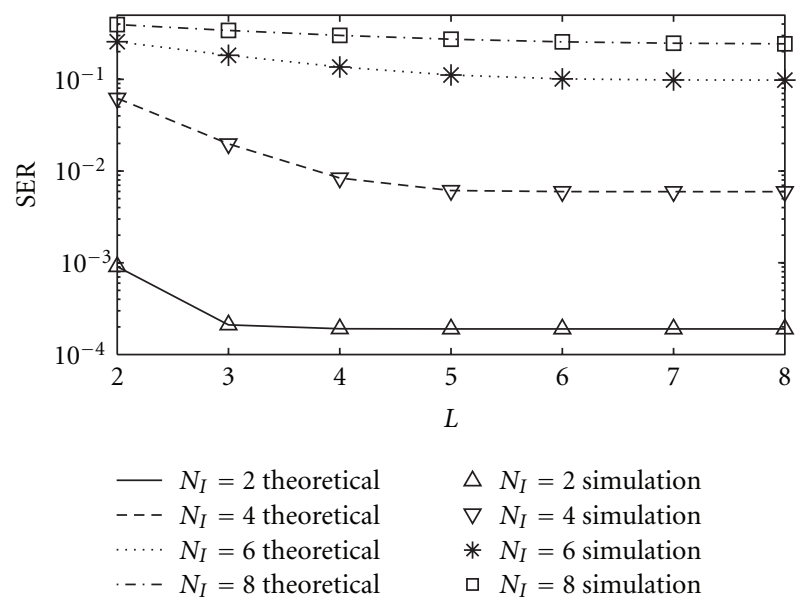

FIgURE 4: SER versus $L$ with different values of $N_{I}$ when $K=6$ and $\mathrm{SIR}=0 \mathrm{~dB}$, and SNR $=10 \mathrm{~dB}$.

SNR, the SER increases as the number of the interferers $N_{I}$ increases and (2) when $N_{I}<L, N_{I}<K$, for example, $N_{I}=$ 2 or 3, the performance of the system is satisfactory, but the performance of the system is the worst while $N_{I}>L, N_{I}>K$.

We plot the SER versus the number of the relay nodes $K$ with SIR $=0 \mathrm{~dB}, \mathrm{SNR}=10 \mathrm{~dB}$ for $L=6$ in Figure 3. In this figure, we consider $N_{I}=2,4,6,8$. From this figure, it can be shown that: (1) for a given working SNR, the more relays there are, the smaller the SER is and (2) for a given $K$, when $L>N_{I}$, the SER is smaller than that when $L \leq N_{I}$, and the space between these two kinds of curves (e.g., $N_{I}=2,4$ and $N_{I}=6,8$ ) becomes wider when the number of the relays becomes larger. That is to say, the increment of the number of the relay nodes can improve the SER performance of system with multiple interferers. Especially when the number of the relay antennas is large enough to combat the interferers at the relay node (i.e., $L>N_{I}$ ), the effect of increasing the number

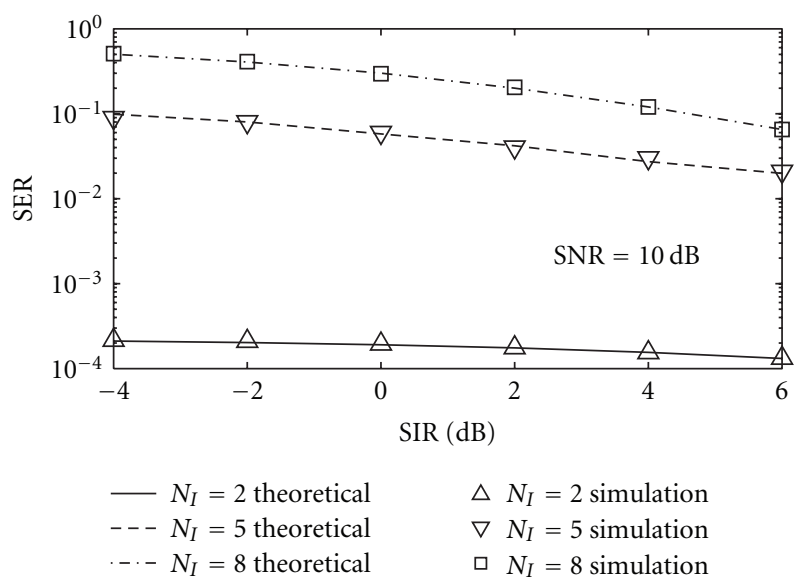

FIGURE 5: SER versus SIR with different values of $N_{I}$ when $L=4$, $K=6$, QPSK interferers.
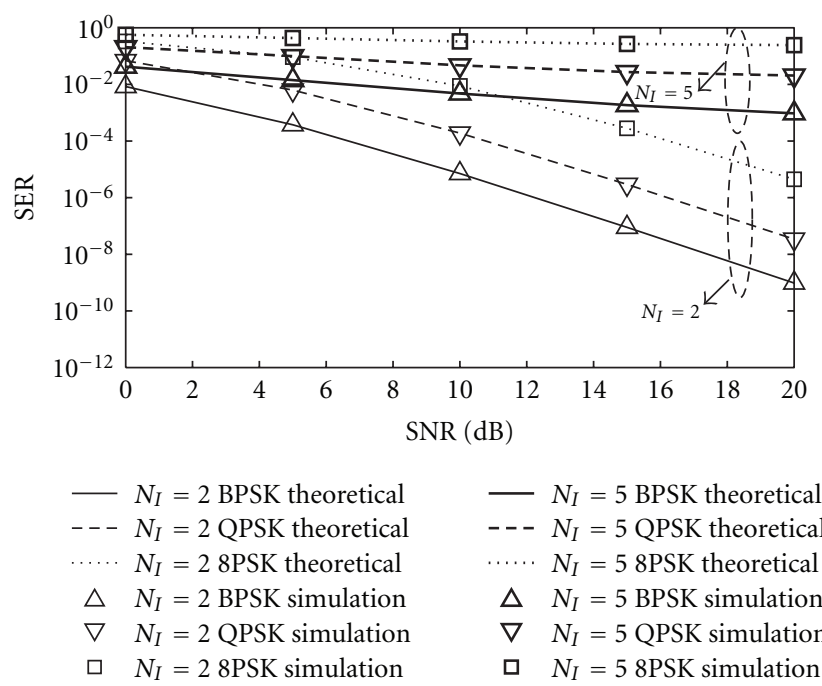

$$
\begin{array}{cc}
- & N_{I}=5 \text { BPSK theoretical } \\
--- & N_{I}=5 \text { QPSK theoretical } \\
\cdots \cdots & N_{I}=5 \text { 8PSK theoretical } \\
\Delta & N_{I}=5 \text { BPSK simulation } \\
\nabla & N_{I}=5 \text { QPSK simulation } \\
\square & N_{I}=5 \text { 8PSK simulation }
\end{array}
$$

FIGURE 6: SER versus SNR with different kinds of modulation when $L=4, K=6$.

of the relay nodes to combat the interferers will become more effective.

The variation of the SER versus the number of the relay antennas $L$ with SIR $=0 \mathrm{~dB}$, SNR $=10 \mathrm{~dB}$ for $K=6$ is shown in Figure 4. We find that: (1) for a given SNR, the SER decreases as $L$ increases whether $N_{I}$ is equal to any value and (2) when the number of the relay antennas $L$ is bigger than a threshold value, the SER performance will be close to each other rather than decreasing evidently even if $L$ increases. It is mainly because that when $L$ is bigger than $N_{I}$, the SER of the $k$ th relay will be smaller, while the probability of properly decoding at the $k$ th relay will become higher. It is easy for us to understand that when the probability of properly decoding is high enough, the set of the selected relays in the system will be almost the same. That is to say, the diversity order of the destination node is almost the same, so the SER performance at the destination node will be close to each other. 
Combining Figures 3 and 4, we can get the fact that increasing the number of the relay nodes, and the number of both relay antennas can improve the SER performance of the system because of increasing diversity order, but the effect of the former is more evident. That is to say, the number of the relay nodes affects the diversity order of the system more greatly. From Figures 3 and 4, we find that the diversity order can combat the interferers effectively, so if we can not utilize enough numbers of the relays and relay antennas simultaneously, it had better to utilize as many as possible relay nodes to increase the diversity order of the system for improving the SER performance.

In Figure 5, we plot the SER versus average SIR per branch $P_{S} / P_{I}$ with SNR $=10 \mathrm{~dB}, L=4$ and $K=6$, using different values of $N_{I}$, and the interference signals are generated as QPSK symbols. From this figure, it can be seen that: (1) although the interference signals are not Gaussian distributed, the simulation results are still very close to the theoretical results. That is to say, the Gaussian assumption for the interference which is necessary for obtaining the theoretical results is not critical for the accuracy of the SER expressions. A similar conclusion was drawn in [30]. In fact, it is well known that OC maximizes the SINR, irrespective of the density function governing the interference [30]; (2) it is obvious that the increase of SIR can improve the SER performance of the system. Although the SER increases with the increase of $N_{I}$ for a given SIR, the more interferers there are in the system, the faster the rate at which the SER falls with the increase in SIR is.

The SER performances of the system versus SNR with $M$-PSK $(M=2,4,8)$ modulation are presented in Figure 6 . Among BPSK, QPSK, and 8PSK modulation modes, the SER performance of 8PSK is worst while that of BPSK is best.

\section{Conclusion}

In this paper, we consider an OFDM-based multiple DF relays network over frequency-selective Rayleigh fading channels and derive an unified closed-form expression of average SER for $M$-PSK modulation in the presence of multiple interferers. Monte-Carlo simulations are carried out to verify our theoretical results.

In the practical OFDM-based relay network, the correlation among the subcarrier channels is unavoidable, and the performance analysis becomes more difficult. Hence, we consider it as a future work.

\section{Appendices}

\section{A. $\varphi_{m}(a, b)$}

$\varphi_{m}(a, b)$ is defined as follows:

$$
\begin{aligned}
\varphi_{m}(a, b)= & \int_{0}^{\infty} \frac{\lambda_{I_{k}}^{m}(n) \exp \left(-\lambda_{I_{k}}(n)\right)}{\sqrt{\lambda_{I_{k}}(n)+a}} \\
& \times \arctan \left(\frac{b}{\sqrt{\lambda_{I_{k}}(n)+a}}\right) d \lambda_{I_{k}}(n), \\
a & >0, \quad m=0,1,2, \ldots .
\end{aligned}
$$

In order to evaluate $\varphi_{m}(a, b)$, we first change the variable of integration to $v=\lambda_{I_{k}}(n)+a$, so

$$
\varphi_{m}(a, b)=\int_{a}^{\infty} \frac{(v-a)^{m} \exp (a-v)}{\sqrt{v}} \arctan \left(\frac{b}{\sqrt{v}}\right) d v .
$$

Using the binomial expansion of $(v-a)^{m}$, (A.2) can be rewritten as

$$
\begin{aligned}
\varphi_{m}(a, b)= & \exp (a) \sum_{i=0}^{m}\left(\begin{array}{c}
m \\
i
\end{array}\right)(-a)^{m-i} \\
& \times \int_{a}^{\infty} v^{i-1 / 2} \exp (-v) \arctan \left(b v^{-1 / 2}\right) d v .
\end{aligned}
$$

Now, let us focus on the evaluation of $R_{i}(a, b)$ which is defined as follows:

$$
R_{i}(a, b)=\int_{a}^{\infty} v^{i-1 / 2} \exp (-v) \arctan \left(b v^{-1 / 2}\right) d v .
$$

Integrating by parts, we obtain the following expression from (A.4):

$$
\begin{aligned}
R_{i}(a, b)= & a^{i-1 / 2} \arctan \left(b a^{-1 / 2}\right) \exp (-a) \\
& +\left(i-\frac{1}{2}\right) \int_{a}^{\infty} \exp (-v) v^{i-3 / 2} \arctan \left(b v^{-1 / 2}\right) d v \\
& -\frac{b}{2} \int_{a}^{\infty} \exp (-v) \frac{v^{i-2}}{1+b^{2} v^{-1}} d v \\
= & a^{i-1 / 2} \arctan \left(b a^{-1 / 2}\right) \exp (-a) \\
& +\left(i-\frac{1}{2}\right) R_{i-1}(a, b)-\frac{b}{2} F_{i}(a, b)
\end{aligned}
$$

where $F_{i}(a, b)=\int_{a}^{\infty} \exp (-v)\left(v^{i-2} /\left(1+b^{2} v^{-1}\right)\right) d v$.

Now, our task is to obtain the closed-form expression of $F_{i}(a, b)$. Because

$$
\begin{aligned}
F_{i}(a, b)= & \int_{a}^{\infty} \exp (-v) \frac{v^{i-1}}{v+b^{2}} d v \\
= & \sum_{j=2}^{i}(-1)^{j-2} b^{2(j-2)} \int_{a}^{\infty} \exp (-v) v^{i-j} d v \\
& +(-1)^{i-1} b^{2(i-1)} \int_{a}^{\infty} \frac{\exp (-v)}{v+b^{2}} d v
\end{aligned}
$$

With the help of $[31,8.350 .2 .11], \int_{a}^{\infty} \exp (-v) v^{i-j} d v$ in (A.6) can be expressed as

$$
\int_{a}^{\infty} \exp (-v) v^{i-j} d v=\Gamma(i-j+1, a) .
$$

And with the help of $[31,3.352 .2], \int_{a}^{\infty}\left(\exp (-v) /\left(v+b^{2}\right)\right) d v$ in (A.6) can be expressed as

$$
\int_{a}^{\infty} \frac{\exp (-v)}{v+b^{2}} d v=-e^{b^{2}} E i\left(-a-b^{2}\right) .
$$


Thus, (A.6) can be rewritten as

$$
\begin{aligned}
F_{i}(a, b)= & \sum_{j=2}^{i}(-1)^{j-2} b^{2(j-2)} \Gamma(i-j+1, a) \\
& -(-1)^{i-1} b^{2(i-1)} e^{b^{2}} E i\left(-a-b^{2}\right) .
\end{aligned}
$$

After obtaining the closed-form expression of $F_{i}(a, b)$, we can solve the difference equation (A.5) to yield [32]

$$
\begin{aligned}
R_{i}(a, b)= & \frac{\Gamma(i+0.5)}{\Gamma(0.5)} R_{0}(a, b)+\arctan \left(b a^{-1 / 2}\right) \exp (-a) \\
& \times \sum_{p=1}^{i} \frac{\Gamma(i+0.5)}{\Gamma(p+0.5)} a^{p-1 / 2} \\
& -\frac{b}{2} \sum_{p=1}^{i} \frac{\Gamma(i+0.5)}{\Gamma(p+0.5)} F_{p}(a, b),
\end{aligned}
$$

where $\Gamma(\cdot)$ denotes the gamma function, and $R_{0}(a, b)$, which is the value of (A.4) when $i=0$, can be calculated beforehand and used as the known constant value.

Substituting (A.9) and (A.10) into (A.3), we can obtain the closed-form expression for $\varphi_{m}(a, b)$

$$
\varphi_{m}(a, b)=\exp (a) \sum_{i=0}^{m}\left(\begin{array}{l}
m \\
i
\end{array}\right)(-a)^{m-i} R_{i}(a, b) .
$$

B. $P_{k, n}(e)$

Let

$$
\begin{aligned}
I_{L-N_{I}} & \left(\frac{(M-1) \pi}{M} ; c_{1}, c_{2}\right) \\
= & \frac{1}{\pi} \int_{0}^{(M-1) \pi / M}\left(\frac{\sin ^{2} \theta}{\sin ^{2} \theta+\left(g_{\mathrm{PSK}} P_{S} \sigma_{S R_{k}}^{2} / \sigma^{2}\right)}\right)^{L-N_{I}} \\
& \times\left(\frac{\sin ^{2} \theta}{\sin ^{2} \theta+\left(g_{\mathrm{PSK}} P_{S} \sigma_{S R_{k}}^{2} /\left(P_{I} \sigma_{I R_{k}}^{2} \lambda_{I_{k}, l}(n)+\sigma^{2}\right)\right)}\right) d \theta,
\end{aligned}
$$

where $c_{1}=g_{\mathrm{PSK}} P_{S} \sigma_{S R_{k}}^{2} / \sigma^{2}, c_{2}=g_{\mathrm{PSK}} P_{S} \sigma_{S R_{k}}^{2} /\left(P_{I} \sigma_{I R_{k}}^{2} \lambda_{I_{k}, l}(n)+\right.$ $\left.\sigma^{2}\right)$, using the same technique as in the case of $N_{I} \geq L$, we can get the following expression:

$$
\begin{aligned}
P_{k, n}(e) & =\frac{1}{N_{I}\left[\prod_{l=1}^{N_{I}}\left(N_{I}-l\right) !(L-l) !\right]} \\
& \times \sum_{l=1}^{N_{I}} \int_{0}^{\infty} \lambda_{I_{k}, l}^{L-N_{I}}(n) \exp \left(-\lambda_{I_{k}, l}(n)\right) \\
& \times I_{L-N_{I}}\left(\frac{(M-1) \pi}{M} ; c_{1}, c_{2}\right)\left[\sum_{p=0}^{N_{I}-1} \beta_{p} \lambda_{I_{k}, l}^{p}(n)\right] d \lambda_{I_{k}, l}(n),
\end{aligned}
$$

where $\beta_{p}\left(p=0,1, \ldots, N_{I}-1\right)$ are the same form as (26) with $L$ and $N_{I}$ exchanged.

From [21, 5A.17] and [21, 5A.56], we know that

$$
\begin{aligned}
& I_{L-N_{I}}\left(\frac{(M-1) \pi}{M} ; c_{1}\right) \\
& =\frac{1}{\pi} \int_{0}^{(M-1) \pi / M}\left(\frac{\sin ^{2} \theta}{\sin ^{2} \theta+c_{1}}\right)^{L-N_{I}} d \theta \\
& =\frac{(M-1)}{M}-\frac{1}{\pi} \sqrt{\frac{c_{1}}{1+c_{1}}} \\
& \times\left\{\left(\frac{\pi}{2}+\arctan \alpha\right) \times \sum_{p=0}^{m-1}\left(\begin{array}{c}
2 p \\
p
\end{array}\right) \frac{1}{\left(4\left(1+c_{1}\right)\right)^{p}}\right. \\
& +\sin (\arctan \alpha) \times \sum_{p=1}^{m-1} \sum_{t=1}^{p} \frac{T_{\mathrm{tp}}}{\left(1+c_{1}\right)^{p}} \\
& \left.\times[\cos (\arctan \alpha)]^{2(p-t)+1}\right\}, \\
& I_{L-N_{I}}\left(\frac{(M-1) \pi}{M} ; c_{1}, c_{2}\right) \\
& =I_{L-N_{I}}\left(\frac{(M-1) \pi}{M} ; c_{1}\right)-\frac{T_{2}}{\pi} \sqrt{\frac{c_{2}}{1+c_{2}}}\left(\frac{c_{2}}{c_{2}-c_{1}}\right)^{L-N_{I}} \\
& +\frac{T_{1}}{\pi} \sqrt{\frac{c_{1}}{1+c_{1}}} \sum_{q=0}^{L-N_{I}-1}\left(\frac{c_{2}}{c_{2}-c_{1}}\right)^{L-N_{I}-q}\left(\begin{array}{c}
2 q \\
q
\end{array}\right) \\
& \times \frac{1}{\left[4\left(1+c_{1}\right)\right]^{q}}+\frac{2}{\pi} \sqrt{\frac{c_{1}}{1+c_{1}}} \sum_{q=0}^{L-N_{I}-1} \sum_{t=0}^{q-1}\left(\frac{c_{2}}{c_{2}-c_{1}}\right)^{L-N_{I}-q} \\
& \times\left(\begin{array}{c}
2 q \\
t
\end{array}\right) \times \frac{(-1)^{t+q}}{\left[4\left(1+c_{1}\right)\right]^{q}} \frac{\sin \left[(2 q-2 t) T_{1}\right]}{2 q-2 t},
\end{aligned}
$$

where $T_{t p}=\left(\begin{array}{c}2 p \\ p\end{array}\right) /\left(\left(\begin{array}{c}2(p-t) \\ p-t\end{array}\right) 4^{t}[2(p-t)+1]\right), \alpha=$ $\sqrt{c_{1} /\left(1+c_{1}\right)} \cot (\pi / M), T_{p}=(1 / 2) \arctan \left(N_{p} / D_{p}\right)+(\pi / 2)[1-$ $\left.\operatorname{sgn}\left(N_{p}\right)\left(\left(1+\operatorname{sgn}\left(D_{p}\right)\right) / 2\right)\right], N_{p}=2 \sqrt{c_{p}\left(1+c_{p}\right)} \sin 2 \phi, D_{p}=$ $\left(1+2 c_{p}\right) \cos 2 \phi-1, p=1,2, \phi=(M-1) \pi / M$. 
Substituting (B.3) into (B.2), we can obtain the following expression:

$$
\begin{aligned}
P_{k, n}(e)= & \frac{1}{\left[\prod_{l=1}^{N_{I}}\left(N_{I}-l\right) !(L-l) !\right]} \\
& \times \sum_{p=0}^{N_{I}-1} \beta_{p}\left\{I_{L-N_{I}}\left(\frac{(M-1) \pi}{M} ; c_{1}\right)\left(L-N_{I}+p\right) !\right. \\
& +\frac{T_{1}}{\pi} \sqrt{\frac{c_{1}}{1+c_{1}}} \sum_{q=0}^{L-N_{I}-1}\left(\begin{array}{c}
2 q \\
q
\end{array}\right) \\
& \times \frac{(q+p) !}{\left[4\left(1+c_{1}\right)\right]^{q}}\left(-\frac{\sigma^{2}}{P_{I} \sigma_{I R_{k}}^{2}}\right) \\
& +\frac{2}{\pi} \sqrt{\frac{c_{1}}{1+c_{1}}} \sum_{q=0}^{L-N_{I}-1} \sum_{t=0}^{q-1}(2 q) \frac{(-1)^{t+q}(q+p) !}{\left[4\left(1+c_{1}\right)\right]^{q}} \\
& \left.\times\left(\frac{\sigma^{2}}{P_{I} \sigma_{I R_{k}}^{2}}\right)^{L-N_{I}} d \lambda_{I_{k}, l}(n)\right\} . \\
& \times \frac{\int_{I_{k}, l}(n)+\left(\left(g_{\mathrm{PSK}} P_{S} \sigma_{S R_{k}}^{2}+\sigma^{2}\right) / P_{I} \sigma_{I R_{k}}^{2}\right)}{2 q-2 t}(n) \exp \left(-\lambda_{I_{k}, l}(n)\right) \frac{T_{2}}{\pi} \sqrt{\frac{g_{\mathrm{PSK}} P_{S} \sigma_{S R_{k}}^{2}}{P_{I} \sigma_{I R_{k}}^{2}}} \\
& \left.\frac{\sigma^{2}}{P_{I} \sigma_{I R_{k}}^{2}}\right)^{L-N_{I}-q}
\end{aligned}
$$$$
\begin{gathered}
=\arctan \left(\frac{(\cos 2 \phi-1) \sqrt{\lambda_{I_{k}, l}(n)+\left(\left(g_{\mathrm{PSK}} P_{S} \sigma_{S R_{k}}^{2}+\sigma^{2}\right) / P_{I} \sigma_{I R_{k}}^{2}\right)}}{2 \sin 2 \phi \sqrt{g_{\mathrm{PSK}} P_{S} \sigma_{S R_{k}}^{2} / P_{I} \sigma_{I R_{k}}^{2}}}\right. \\
\left.+\frac{\sqrt{g_{\mathrm{PSK}} P_{S} \sigma_{S R_{k}}^{2} / P_{I} \sigma_{I R_{k}}^{2}}(\cos 2 \phi+1)}{2 \sin 2 \phi \sqrt{\lambda_{I_{k}, l}(n)+\left(\left(g_{\mathrm{PSK}} P_{S} \sigma_{S R_{k}}^{2}+\sigma^{2}\right) / P_{I} \sigma_{I R_{k}}^{2}\right)}}\right) .
\end{gathered}
$$

Let

$$
\begin{gathered}
x=\frac{(\cos 2 \phi-1) \sqrt{\lambda_{I_{k}, l}(n)+\left(\left(g_{\mathrm{PSK}} P_{S} \sigma_{S R_{k}}^{2}+\sigma^{2}\right) / P_{I} \sigma_{I R_{k}}^{2}\right)}}{2 \sin 2 \phi \sqrt{g_{\mathrm{PSK}} P_{S} \sigma_{S R_{k}}^{2} / P_{I} \sigma_{I R_{k}}^{2}}}, \\
y=\frac{\sqrt{g_{\mathrm{PSK}} P_{S} \sigma_{S R_{k}}^{2} / P_{I} \sigma_{I R_{k}}^{2}}(\cos 2 \phi+1)}{2 \sin 2 \phi \sqrt{\lambda_{I_{k}, l}(n)+\left(\left(g_{\mathrm{PSK}} P_{S} \sigma_{S R_{k}}^{2}+\sigma^{2}\right) / P_{I} \sigma_{I R_{k}}^{2}\right)}},
\end{gathered}
$$

we can find that

$$
\begin{gathered}
x y=\frac{\cos ^{2} 2 \phi-1}{4 \sin ^{2} 2 \phi}=-\frac{1}{4}<1, \\
\frac{2 x+2 y}{1-2 x \cdot 2 y}=x+y .
\end{gathered}
$$

With the help of $[31,1.625 .8]$, (C.2) can be expressed as

$$
\begin{aligned}
\arctan \left(\frac{D_{2}}{N_{2}}\right) & =\arctan (x+y)=\arctan \left(\frac{2 x+2 y}{1-2 x \cdot 2 y}\right) \\
& =\arctan (2 x)+\arctan (2 y) \\
& =\frac{\pi}{2}-\arctan \left(\frac{1}{2 x}\right)+\arctan (2 y) .
\end{aligned}
$$

$\left.2 c_{2}\right) \cos 2 \phi-1, \phi=(M-1) \pi / M$, we can rewritten $T_{2}$ into another form as

$$
\begin{aligned}
T_{2}= & \frac{1}{2} \operatorname{sgn}\left(\operatorname{sgn}\left(\frac{N_{2}}{D_{2}}\right)+\frac{1}{2}\right) \times \frac{\pi}{2}-\frac{1}{2} \arctan \left(\frac{D_{2}}{N_{2}}\right) \\
& +\frac{\pi}{2}\left[1-\operatorname{sgn}\left(N_{2}\right) \frac{1+\operatorname{sgn}\left(D_{2}\right)}{2}\right] .
\end{aligned}
$$

Now, our task is to simplify the expression of $\arctan \left(D_{2} / N_{2}\right)$. Because

$$
\begin{aligned}
& \arctan \left(\frac{D_{2}}{N_{2}}\right) \\
& =\arctan \frac{\left(1+2 c_{2}\right) \cos 2 \phi-1}{2 \sqrt{c_{2}\left(1+c_{2}\right)} \sin 2 \phi}
\end{aligned}
$$

Thus, (C.1) can be rewritten as

$$
\begin{aligned}
T_{2}= & \frac{\pi}{2}\left[\frac{1}{2} \operatorname{sgn}\left(\operatorname{sgn}\left(\frac{N_{2}}{D_{2}}\right)+\frac{1}{2}\right)+1-\operatorname{sgn}\left(N_{2}\right) \frac{1+\operatorname{sgn}\left(D_{2}\right)}{2}\right] \\
& -\frac{1}{2}\left(\frac{\pi}{2}-\arctan \left(\frac{1}{2 x}\right)+\arctan (2 y)\right) \\
= & \frac{\pi}{2}\left[\frac{1}{2} \operatorname{sgn}\left(\operatorname{sgn}\left(\frac{N_{2}}{D_{2}}\right)+\frac{1}{2}\right)+\frac{1}{2}-\operatorname{sgn}\left(N_{2}\right) \frac{1+\operatorname{sgn}\left(D_{2}\right)}{2}\right] \\
& +\frac{1}{2} \arctan \left(\frac{1}{2 x}\right)-\frac{1}{2} \arctan (2 y) .
\end{aligned}
$$


Substituting (C.6) into (32), we can obtain the closed-form expression of $B$

$$
\begin{aligned}
& B=-\frac{1}{2}\left[\frac{1}{2} \operatorname{sgn}\left(\operatorname{sgn}\left(\frac{N_{2}}{D_{2}}\right)+\frac{1}{2}\right)+\frac{1}{2}\right. \\
& \left.-\operatorname{sgn}\left(N_{2}\right) \frac{1+\operatorname{sgn}\left(D_{2}\right)}{2}\right] \\
& \times \sqrt{\frac{g_{\mathrm{PSK}} P_{S} \sigma_{S R_{k}}^{2}}{P_{I} \sigma_{I R_{k}}^{2}}} g_{p}\left(\frac{g_{\mathrm{PSK}} P_{S} \sigma_{S R_{k}}^{2}+\sigma^{2}}{P_{I} \sigma_{I R_{k}}^{2}}\right)\left(-\frac{\sigma^{2}}{P_{I} \sigma_{I R_{k}}^{2}}\right)^{L-N_{I}} \\
& -\frac{1}{2 \pi} \sqrt{\frac{g_{\mathrm{PSK}} P_{S} \sigma_{S R_{k}}^{2}}{P_{I} \sigma_{I R_{k}}^{2}}}\left(-\frac{\sigma^{2}}{P_{I} \sigma_{I R_{k}}^{2}}\right)^{L-N_{I}} \\
& \times \varphi_{p}\left(\frac{g_{\mathrm{PSK}} P_{S} \sigma_{S R_{k}}^{2}+\sigma^{2}}{P_{I} \sigma_{I R_{k}}^{2}}, \frac{\sin 2 \phi}{\cos 2 \phi-1} \sqrt{\frac{g_{\mathrm{PSK}} P_{S} \sigma_{S R_{k}}^{2}}{P_{I} \sigma_{I R_{k}}^{2}}}\right) \\
& +\frac{1}{2 \pi} \sqrt{\frac{g_{\mathrm{PSK}} P_{S} \sigma_{S R_{k}}^{2}}{P_{I} \sigma_{I R_{k}}^{2}}}\left(-\frac{\sigma^{2}}{P_{I} \sigma_{I R_{k}}^{2}}\right)^{L-N_{I}} \\
& \times \varphi_{p}\left(\frac{g_{\mathrm{PSK}} P_{S} \sigma_{S R_{k}}^{2}+\sigma^{2}}{P_{I} \sigma_{I R_{k}}^{2}}, \frac{\cos 2 \phi+1}{\sin 2 \phi} \sqrt{\frac{g_{\mathrm{PSK}} P_{S} \sigma_{S R_{k}}^{2}}{P_{I} \sigma_{I R_{k}}^{2}}}\right) .
\end{aligned}
$$

\section{Acknowledgments}

This work is supported by the National Natural Science Foundation of China under Grant no. 60972051, the Jiangsu Province Natural Science Foundation of China under Grant no. BK2010101, the Open Research Fund of National Mobile Communications Research Laboratory, Southeast University under Grant no. 2010D09 and the Important National Science \& Technology Specific Project under Grant no. 2010ZX03006-002-04.

\section{References}

[1] A. Sendonaris, E. Erkip, and B. Aazhang, "User cooperation diversity-part I: system description," IEEE Transactions on Communications, vol. 51, no. 11, pp. 1927-1938, 2003.

[2] J. N. Laneman, D. N. C. Tse, and G. W. Wornell, "Cooperative diversity in wireless networks: efficient protocols and outage behavior," IEEE Transactions on Information Theory, vol. 50, no. 12, pp. 3062-3080, 2004.

[3] A. Nosratinia, T. E. Hunter, and A. Hedayat, "Cooperative communication in wireless networks," IEEE Communications Magazine, vol. 42, no. 10, pp. 74-80, 2004.

[4] M. O. Hasna and M.-S. Alouini, "End-to-end performance of transmission systems with relays over Rayleigh-fading channels," IEEE Transactions on Wireless Communications, vol. 2, no. 6, pp. 1126-1131, 2003.

[5] M. O. Hasna and M.-S. Alouini, "Outage probability of multihop transmission over Nakagami fading channels," IEEE Communications Letters, vol. 7, no. 5, pp. 216-218, 2003.

[6] K. G. Seddik, A. K. Sadek, W. Su, and K. J. R. Liu, "Outage analysis and optimal power allocation for multinode relay networks," IEEE Signal Processing Letters, vol. 14, no. 6, pp. 377-380, 2007.
[7] V. A. Aalo and J. Zhang, "Performance of antenna array systems with optimum combining in a Rayleigh fading environment," IEEE Communications Letters, vol. 4, no. 4, pp. 125-127, 2000.

[8] A. Shah, A. M. Haimovich, M. K. Simon, and M.-S. Alouini, "Exact bit-error probability for optimum combining with a rayleigh fading Gaussian cochannel interférer," IEEE Transactions on Communications, vol. 48, no. 6, pp. 908-912, 2000.

[9] E. Villier, "Performance analysis of optimum combining with multiple interferers in flat Rayleigh fading," IEEE Transactions on Communications, vol. 47, no. 10, pp. 1503-1510, 1999.

[10] Y. Zhu and H. Zheng, "Understanding the impact of interference on collaborative relays," IEEE Transactions on Mobile Computing, vol. 7, no. 6, pp. 724-735, 2008.

[11] A. Agustin and J. Vidal, "Amplify-and-forward cooperation under interference-limited spatial reuse of the relay slot," IEEE Transactions on Wireless Communications, vol. 7, no. 5, pp. 1952-1962, 2008.

[12] C. Zhong, S. Jin, and K.-K. Wong, "Dual-hop systems with noisy relay and interference-limited destination," IEEE Transactions on Communications, vol. 58, no. 3, pp. 764-768, 2010.

[13] G. Li and H. Liu, "Resource allocation for OFDMA relay networks with fairness constraints," IEEE Journal on Selected Areas in Communications, vol. 24, no. 11, pp. 2061-2068, 2006.

[14] L. Dai, B. Gui, and L. J. Cimini Jr., "Selective relaying in OFDM multihop cooperative networks," in Proceedings of the IEEE Wireless Communications and Networking Conference (WCNC '07), pp. 964-969, Kowloon, HongKong, March 2007.

[15] B. Gui, L. Dai, and L. J. Cimini Jr., "Selective relaying in cooperative OFDM systems: two-hop random network," in Proceedings of the IEEE Wireless Communications and Networking Conference (WCNC '08), pp. 996-1001, Las Vegas, Nev, USA, April 2008.

[16] R. K. Mallik, M. Z. Win, M. Chiani, and A. Zanella, "Biterror probability for optimum combining of binary signals in the presence of interference and noise," IEEE Transactions on Wireless Communications, vol. 3, no. 2, pp. 395-407, 2004.

[17] A. Adinoyi and H. Yanikomeroglu, "Cooperative relaying in multi-antenna fixed relay networks," IEEE Transactions on Wireless Communications, vol. 6, no. 2, pp. 533-544, 2007.

[18] H. Katiyar and R. Bhattacharjee, "Performance of two-hop regenerative relay network under correlated nakagami-m fading at multi-antenna relay," IEEE Communications Letters, vol. 13, no. 11, pp. 820-822, 2009.

[19] T. Keller and L. Hanzo, "Adaptive modulation techniques for duplex OFDM transmission," IEEE Transactions on Vehicular Technology, vol. 49, no. 5, pp. 1893-1906, 2000.

[20] M. Chiani, M. Z. Win, and A. Zanella, "Error probability for optimum combining of M-ary PSK signals in the presence of interference and noise," IEEE Transactions on Communications, vol. 51, no. 11, pp. 1949-1957, 2003.

[21] M. K. Simon and M. S. Alouini, Digital Communication over Fading Channels, John Wiley \& Sons, New York, NY, USA, 2nd edition, 2005.

[22] W. W. Peterson and E. J. Weldon, Error Correcting Codes, MIT Press, Cambridge, Mass, USA, 1972.

[23] H. Bölcskei, D. Gesbert, and A. J. Paulraj, "On the capacity of OFDM-based spatial multiplexing systems," IEEE Transactions on Communications, vol. 50, no. 2, pp. 225-234, 2002.

[24] S. Kaiser, "OFDM-CDMA versus DS-CDMA: performance evaluation for fading channels," in Proceedings of the IEEE International Conference on Communications (ICC '95), pp. 1722-1726, June 1995. 
[25] X. D. Zhang, Matrix Analysis and Applications, Tsinghua University Press, Beijing, China; Springer, Berlin, Germany, 2004.

[26] J. G. Proakis, Digital Communications, Publishing House of Electronics Industry, 4th edition, 2006.

[27] L. Zheng and D. N. C. Tse, "Diversity and multiplexing: a fundamental tradeoff in multiple-antenna channels," IEEE Transactions on Information Theory, vol. 49, no. 5, pp. 10731096, 2003.

[28] Z. Wang and G. B. Giannakis, "A simple and general parameterization quantifying performance in fading channels," IEEE Transactions on Communications, vol. 51, no. 8, pp. 13891398, 2003.

[29] Z. Kang and K. Yao, "On the performances of MC-CDMA over frequency-selective nakagami-m fading channels with correlated and independent subcarriers," in Proceedings of the IEEE Global Telecommunications Conference (GLOBECOM '04), vol. 5, pp. 2859-2863, Dallas, Tex, USA, NovemberDecember 2004.

[30] D. Lao and A. M. Haimovich, "Exact closed-form performance analysis of optimum combining with multiple cochannel interferers and Rayleigh fading," IEEE Transactions on Communications, vol. 51, no. 6, pp. 995-1003, 2003.

[31] I. S. Gradsheteyn and I. M. Ryzhik, Table of Integrals, Series and Products, Academic, San Diego, Calif, USA, 1980.

[32] R. K. Mallik, "Solutions of linear difference equations with variable coefficients," Journal of Mathematical Analysis and Applications, vol. 222, no. 1, pp. 79-91, 1998. 